\title{
Nucleus Accumbens Dopamine D3 Receptor Regulates Microglial M1 Polarization Through Akt Signaling Pathway in the Depressive-Like Behavior
}

Jing Wang

Xi'an Jiaotong University

Simin Lai

Xi'an Jiaotong University

Rui Wang

Xi'an Jiaotong University

Ting Zhou

Xi'an Jiaotong University Second Affiliated Hospital

Nan Dong

Xi'an Jiaotong University

Li Zhu

Xi'an Jiaotong University

Teng Chen

Xi'an Jiaotong University

Yanjiong Chen ( $\nabla$ chenyanjiong@126.com )

Department of Immunology and Pathogenic Biology, College of Basic Medicine, Xi'an Jiaotong University Health Science Center https://orcid.org/0000-0001-8510-6004

\section{Research}

Keywords: NAc inflammation, D3R, microglia polarization, Akt signaling pathway, depression

Posted Date: May 7th, 2021

DOI: https://doi.org/10.21203/rs.3.rs-433541/v1

License: (c) (i) This work is licensed under a Creative Commons Attribution 4.0 International License.

Read Full License 


\section{Abstract}

Background: Nucleus accumbens (NAc) inflammation is implicated in the development and progression of depression but the underlying mechanism remains largely unclear. We previously reported that dopamine D3 receptor (D3R) expressed in the NAc strongly correlated with inflammation-related depression. The objective of the present study is to determine the exact mechanism of D3R in the NAC inflammation relevant depression.

Methods: Bilateral NAc injection of Lipopolysaccharide (LPS)-induced NAc inflammation mouse model was used to explore the relationship between NAc inflammation and depressive-like behaviors. Either conditional knockout of D3R in the NAc in the adult mice or restoration of D3R expression in the NAc in D3R mutant mice was applied to investigate the effects of D3R in the NAc on depressive disorders. The exact molecular mechanism of D3R in the NAc inflammation-induced depressive-like phenotype was ascertained by in vivo and in vitro studies.

Results: NAc inflammation induced by intra-NAc LPS triggered a series of depressive-like disorders, and the interaction of D3R and microglia activation involved in the NAc inflammation-induced depressive-like phenotype. We observed that selective knock-down of D3R in the NAc elicited the depressive-like behaviors, but re-expression of D3R in the NAc alleviated the depressive-like behaviors established by D3R mutation. Finally, D3R in the NAc mediated microglial polarization to M1 phenotype mainly through Akt signaling pathway.

Conclusions: These findings provide direct evidence that not only NAc inflammation but also downregulation of D3R in the NAc has a role in regulation the initiation and development of depression, and indicate that D3R negatively regulated microglial M1 polarization mainly via Akt signaling pathway that may be involved in the NAc inflammation and thereby depressive-like behaviors.

\section{Background}

Major depression is a prevalent and devastating psychiatric disorder affecting nearly 350 million people worldwide, and has become the world's leading cause of disability and exerts an extraordinary economic burden on society in terms of lost productivity [1, 2]. Indeed, depression leads to an evident decline in lifespan, in part result from suicide, and the remainder result from the significant increase in vulnerability to other chronic illness, such as cardiovascular disease, autoimmune disease, diabetes, and cancer [3, 4]. Unfortunately, due to its complexity and heterogeneity, mechanisms associated with the pathogenesis of depression have yet to be clearly well-understood. And in fact, only half of depressed patients are responsive to currently antidepressant treatments [5]. Therefore, it is urgently needed to in-depth understand the pathogenesis of depression and develop more effective therapies.

Microglia, as the principal resident immune cells of the brain, have received a great deal of attention during the past decades. Under normal conditions, resting microglia characterized by a small soma endowed with several extra-thin but highly motile processes responsible for continuously monitor the 
brain parenchyma to maintain brain homeostasis [6]. But when the response to pathogen stimuli, microglia undergo morphological changes and mainly convert to M1 and M2 phenotypes of activated microglia, accompanied by prominently expressing pro-inflammatory and anti-inflammatory cytokines in the brain [7]. Accumulating recent evidence indicate that microglia sense depression-related stressors and elicit neuroinflammation that subsequently contribute to depression. Post-mortem brain studies have found that the percentage of activated microglia is increased in the dorsal anterior cingulate cortex of depressed suicides compared to those without psychiatric or inflammatory diseases [8]. In line with this phenomenon, positron emission tomography (PET) observed that levels of translocator protein $18 \mathrm{kD}$ (TSPO), which correlates with activated microglia-related neuroinflammation, were significantly enhanced in the prefrontal cortex, anterior cingulate cortex and insula in patients with major depression $[9,10]$. Furthermore, in both inflammatory (LPS) and non-inflammatory (stress) animal models of depression, the microglia in the brain were activated and trigger pro-inflammatory cytokines production, whereas to delete microglia applied by PLX compounds and use anti-inflammatory drug minocycline both exhibited antidepressant effects in rodent models of depression [11-15]. In this regard, activated microglia and itsderived proinflammatory cytokines might directly contribute to the onset of depression.

Dysfunction of dopaminergic neurotransmission within the mesolimbic system, particularly in the nucleus accumbens (NAc), is thought to mediate hedonic deficits, loss of motivation and psychomotor retardation in depressive disorders [16-18]. Importantly, several lines of research have demonstrated that NAc inflammation plays a considerable role in depressive illness. Chronic stress-induced depressive-like phenotype activated microglial cells in the NAc [19]. Likewise, depressive-like behaviors under highfat/high-sucrose diets and systemic LPS injection both facilitated microglia activation in the NAc, as well as enhanced NAc expression of proinflammatory mediators [20,21]. Of note, one clinical study demonstrates that the mood-dependent modulation of NAc in inflammation-associated in total mood might underlie anhedonia, which is a core feature of depression [22]. In spite of NAc inflammation has been strongly implicated in development of depressive abnormality as described above, the causal relationship of NAc inflammation and the establishment of depressive-like symptoms are unknown, and then little is known which types of special molecular could elicit NAc inflammation and subsequent depression.

The dopamine D3 receptor (D3R), which restrictedly expresses in the mesolimbic reward brain areas, has been demonstrated to be the central role in the pathophysiology of depressive disorders [23, 24].

Consistent with previous studies, our published data in rodent model of peripheral inflammation-induced depressive-like behaviors showed that D3R expression in the NAc was reduced but activation of D3R could ameliorate behavioral despair induced by peripheral inflammation [21]. Of interest, we observed that D3R mutation-triggered depressive-like behaviors had a strong correlation with microglia activation in the NAc [25], these results provide a novel possibility of D3R in the inflammation relevant depression. Importantly, current studies in the depression highlight potential therapeutic effect of D3R. For example, clinical reports in depressed patients demonstrate that genetic variation in the $D 3 R$ gene can affect the response to selective serotonin reuptake inhibitors (SSRIs), such as paroxetine [26]. And several experimental drugs in pre-clinical or clinical development may act as antidepressants due to their partial 
agonist activity at D3R [27], suggesting efforts in finding the exact mechanism of D3R in depression seems presently significant.

Based on currently available data, we plan to uncover the effects of D3R expressed in the NAc on the inflammation relevant depression. First, we will determine the causal relationship between NAC inflammation and depressive-like behaviors, and the correlation of D3R and microglia activation in the NAc under NAc inflammation condition. Thereafter, we will then discuss the possible mechanism on how D3R regulates NAc inflammation-associated depressive-like behaviors. Understanding the D3R-related mechanism in inflammation-induced depressive-like phenotype will shed light on developing new therapeutic strategies to treat this prevalent disease.

\section{Methods}

\section{Animals}

All experimental procedures were performed in compliance with the National Institutes of Health guidelines for the use of experimental animals and approved by the Institutional Animal Care and Use Committee of Xi'an Jiaotong University. Mice were single housed in a temperature- and humiditycontrolled room with a 12-h reverse light/dark cycle and provided ad libitum access to water and food. 7week-old male C57BL/6 mice were purchased from the Beijing Vital River Laboratory Animal Technology Co. Ltd and allowed one week of acclimation to the housing conditions before the initiation of experiments. Male dopamine D3 receptor-knockout (D3RKO) mice were a kind gift from Professor Xu (Department of Anaesthesia and Critical Care, The University of Chicago) as described previously [25], and all mice were bred at the housing facilities and used at 8-12 weeks of age. All animals were randomly allocated to the different experimental groups used in this study.

\section{Pharmacological treatments and viral vectors}

Lipopolysaccharide (LPS) from Escherichia coli (L-3129, serotype 0127: B8) was purchased from SigmaAldrich, they were freshly dissolved in sterile saline. Minocycline (a microglial inhibitor), PD128907 (dopamine D3 receptor agonist), MK2206 (Akt signalling pathway inhibitor) and SP600125 (JNK signalling pathway inhibitor) were obtained from Selleckchem (Houston, TX, USA); minocycline and PD128907 were dissolved in $0.9 \%$ saline, and MK2206 and SP600125 were diluted in $0.1 \%$ dimethylsulfoxide (DMSO) they were stored at $-80^{\circ} \mathrm{C}$. Cytokine IL-4 was purchased from Pepro Tech (Rocky Hills, NJ), dissolved into PBS and kept at $-20^{\circ} \mathrm{C}$. The adeno-associated virus (AAV) vector was used to genetically over-express D3R (pAAV-CMV-EGFP-2A-Drd3-3FLAG, AAV-D3R), and AAV expressing U6-driven shRNA and CMV-driven mCherry were used to inhibit D3R (pAAV-U6-shDrd3-CMV-EGFP-WPREspolyA, AAV-shD3R). Their vectors were both serotyped with AAV2/8 and purchased from Obio Technology, and the viral titer respectively was $1 \times 10^{12}$ and $1 \times 10^{13}$ particles per $\mathrm{ml}$. All viral vectors were aliquoted and stored at $-80^{\circ} \mathrm{C}$ until used.

BV-2 microglial cell culture 
The immortalized murine microglial cell line, BV-2 cell, was purchased from the Kunming Institute of Zoology in the Chinese Academy of Sciences (Kunming, China). BV-2 microglial cells were cultured in Dulbecco's modified Eagle's medium (DMEM) supplemented with $10 \%$ fetal bovine serum (FBS) and $1 \%$ penicillin/streptomycin at $37{ }^{\circ} \mathrm{C}$ in a humidified atmosphere containing $95 \%$ air and $5 \% \mathrm{CO}^{2}$. Prior to experimental use, BV-2 microglia were seeded on $100 \mathrm{~mm}$ culture dishes, and the medium was changed every two days. When they reached $70-80 \%$ confluence, the cells were split with $0.25 \%$ trypsin and then seeded on 24-well culture plates $\left(2 \times 10^{4} /\right.$ well) for experimental use. After control siRNA and D3R siRNA transfected into BV-2 microglia, cells were treated with or without LPS $(100 \mathrm{ng} / \mathrm{ml})$ or IL-4 $(20 \mathrm{ng} / \mathrm{ml})$ for $24 \mathrm{~h}$. In the MK2206 and SP600125 treatment groups, BV-2 cells were simultaneously treated with MK2206 $(20 \mu \mathrm{M}$ and $40 \mu \mathrm{M})$ / SP600125 $(80 \mu \mathrm{M}$ and $160 \mu \mathrm{M})$ and LPS for $24 \mathrm{~h}$ after cells were pretreated with small interfering RNA (siRNA).

\section{Primary microglial cell culture}

Primary murine microglia (PMM) were isolated and purified from striatum and mid-brain of neonatal mice (P3-P5) as previously described [28]. In brief, three-five fetal mice brains from WT or D3RKO mice were removed from skull, meninges were stripped and the striatum and mid-brain regions were dissected. Gilal cells were disaggregated by trypsinization ( $0.25 \%$ trypsin, $\left.5 \mathrm{~min}, 37^{\circ} \mathrm{C}, 5 \% \mathrm{CO} 2\right)$, and cells were washed, resuspended in DMEM media supplemented with $10 \%$ FBS and $1 \%$ penicillin/streptomycin. Afterward, the cell suspension was seeded onto $75 \mathrm{~cm}^{2}$ tissue culture flasks precoated with $5 \mathrm{mg} / \mathrm{ml}$ poly-D-lysine at 37 ${ }^{\circ} \mathrm{C}$ in humidified atmosphere of $5 \% \mathrm{CO}_{2}$. After incubation for $24 \mathrm{~h}$, the media was replaced by fresh media. Next, half of the volume of media was renovated every 3-4 days and cells were cultured for additional 10 to 14 days. Microglia were obtained from mixed glial culture by mechanical extraction using a horizontal rotating shaker at 230-250 rpm for $2 \mathrm{~h}$ and seeded onto poly-D-lysine-coated 24-well culture plates $\left(2-3 \times 10^{4} /\right.$ well $)$ for further use. The purity of the microglial cells obtained was evaluated by CD11b immunostaining ( $\geq 95 \%$ CD11 $b^{+}$cells). The primary microglial cells obtained from WT and D3RKO mice were treated with or without LPS $(100 \mathrm{ng} / \mathrm{ml})$ for $24 \mathrm{~h}$.

\section{SiRNA transfection}

D3R expression was transiently silenced using D3R siRNA (XIEBHC Biotechnology Beijing, China) according to the manufacturer's instructions. Briefly, cultured BV-2 cells were plated at a density of $2 \times 10^{4}$ cells per 24 -well plate. After $18 \mathrm{~h}$ of growth to $30 \%-50 \%$ confluence, the cells were transfected with control siRNA (nonspecific scramble siRNA that does not target any mouse genes) or D3R siRNA using a mixture of plasmid and lipofectamine2000 (Invitrogen, Carlsbad, USA) in OPTI-MEN according to manufacturer's specification. The cells were incubated with the siRNA mix for 6 hours and then the medium was replaced with new DMEM media with $10 \% \mathrm{FBS}$ and incubated for another $40 \mathrm{~h}$ at $37^{\circ} \mathrm{C}$ in humidified atmosphere of $5 \% \mathrm{CO}_{2}$ for protein extraction to check the knockdown efficiency by western blot.

\section{Stereotaxic surgery}


Mice were anaesthetized with isoflurane (4.0\% for induction and $1.0 \%$ thereafter for maintenance), and their heads were fixed in a stereotaxic frame (RWD Life Science Co., Shenzhen, China). The skull surface was exposed and all skull measurements were made relative to bregma, drugs or packaged virus injection into the NAc was performed using a microinjection needle with a 10- $\mu$ l microsyringe (Hamilton, USA) to deliver at a rate of $0.15 \mu \mathrm{l} /$ minute using an automated injection pump (KDS Legato ${ }^{\text {TM }} 130$, USA). Then the injection needle was withdrawn $10 \mathrm{~min}$ after the end of the infusion. The stereotaxic coordinates for nucleus accumbens (NAc) injection were anterior posterior (AP) $1.50 \mathrm{~mm}$, medial lateral (ML) $1.00 \mathrm{~mm}$ and dorsal ventral (DV) $4.50 \mathrm{~mm}$ relative to Bregma. In the pharmacological treatments, $0.3 \mu \mathrm{l}$ of minocycline $(8 \mu \mathrm{g})$, PD128907 $(4 \mu \mathrm{g})$ or vehicle $(0.9 \%$ saline) was bilaterally injected into the NAc 30 min prior to saline $(0.3 \mu \mathrm{l})$ or LPS $(0.3 \mu \mathrm{l}, 100 \mathrm{ng})$ administration. The dose of LPS was selected based on its ability to induce depressive-like behaviors that are temporally distinct from acute sickness behaviors [29], and the doses of minocycline and PD128907 were chose based on our preliminary experiment showing the beneficial effects of the minimum dose in an animal model of depressive-like behaviors exposed to NAc administration of LPS. At the end of the experiment, trypan blue solution $(0.4 \%)$ was injected into the $\mathrm{NAc}$, and the injection sites were histologically verified with Nissl staining. For in vivo viral injection, $0.3 \mu \mathrm{l}$ of virus expressing AAV-D3R or AAV-shD3R were infused into the bilateral NAc of the D3RKO or WT mice, respectively. Mice were used at least one week after AAV injection.

\section{Depressive-like behavioral tests}

All tests were carried out during the light phase (between 9:00 am and 4:00 pm), and the mice were allowed to acclimatize to the behavioral test room for at least one hour. The forced swim test (FST) and tail suspension test (TST) were performed after $24 \mathrm{~h}$ of LPS/saline injection or 4 weeks of virus administration. In the sucrose preference test, $24 \mathrm{~h}$ of the water and sucrose consumption were measured after pharmacological and viral treatments. According to our previous study [25], the FST was conducted as follows. Mice were placed in a transparent plastic cylinder (30 cm height $\times 20 \mathrm{~cm}$ diameter) that was partially filled to a height of $15 \mathrm{~cm}$ with $24 \pm 1^{\circ} \mathrm{C}$ water for $6 \mathrm{~min}$. The water in the cylinder was changed between each mouse during the test session. The test duration was video-recorded for $6 \mathrm{~min}$, and the mice were calculated for the final 4 min of immobile time. The TST was conducted as follows. Mice were suspended $30 \mathrm{~cm}$ above the floor by hanging on a fixed hook using a small piece of adhesive tape placed approximately $2 \mathrm{~cm}$ from the tip of the tail for $10 \mathrm{~min}$. The duration of immobility over the $10 \mathrm{~min}$ was recorded and calculated. The sucrose preference test was conducted as follows. To quantify inflammation-induced anhedonia, we subjected mice to the two-bottle sucrose preference test. Approximately 2 weeks prior to treatment, mice were trained by simultaneous presentation with a bottle of water and a bottle of $1 \%(\mathrm{wt} / \mathrm{vol})$ sucrose solution. The bottles were weighed prior to being placed on the lid of each mouse's home cage and reweighed to determine the amount of sucrose solution and water that had been consumed after $24 \mathrm{~h}$. The positions of the bottles were changed every $12 \mathrm{~h}$ to ensure that the mice did not develop a preference for one side. Mice were trained until a stable baseline preference was established, and then, treatments were administered. Sucrose preference was calculated as the percentage of sucrose solution consumed relative to the total fluid intake: sucrose intake/(sucrose intake 
+ water intake) $\times 100$. The behavioral tests were performed by experimenters who were blinded to the experimental groups.

\section{Tissue collection and quantitative real-time RT-PCR (qRT-PCR) analysis}

At $0,4,12$, or $24 \mathrm{~h}$ post-injection of LPS and after 4 weeks of virus injection, mice were euthanized by sodium pentobarbital solution (100 mg/kg). NAc brain tissue was dissected bilaterally on ice and immediately frozen in sample tubes placed in liquid nitrogen. The tissue was stored frozen at $-80^{\circ} \mathrm{C}$ until processing. Next, total RNA was isolated using the TRIzol® RNA extraction kit (Invitrogen). Reverse transcription was performed using $1 \mu \mathrm{g}$ of total RNA for each sample by using the PrimeScriptTM RT reagent kit (Takara Bio Inc.) according to the manufacturer's instructions. Real-time PCR amplification was performed using the Stratagene Mx 3005p Real-Time PCR Detection System (Agilent Technologies, Santa Clara, CA, USA) with SYBR Green master mix (Takara Bio Inc.) in a final volume of $20 \mu \mathrm{L}$ that contained $1 \mu \mathrm{L}$ of cDNA template from each sample. The primers used in our study are listed as follows: D3R forward: CATGATTACGGCTGTGTGGG, D3R reverse: GAGGATCCGTTTTCTTCGCC; Iba1 forward: ACGAACCCTCTGATGTGGTC, Iba1 reverse: TGAGGAGGACTGGCTGACTT; GAPDH forward:

TGTGTCCGTCGTGGATCTGA; GAPDH reverse: TTGCTGTTGAAGTCGCAGGAG. The relative mRNA values were normalized to the control values of the GAPDH gene and calculated using the comparative cycle threshold $(\triangle \triangle \mathrm{Ct})$ method [30].

\section{Western blot analysis}

Western blot was performed as described previously [25]. Briefly, NAc tissue after LPS/saline and cultured BV-2 cells treated with siRNA $24 \mathrm{~h}$ after LPS challenge were collected and lysed in RIPA lysis buffer ( $1 \mathrm{x}$ phosphate-buffered saline, $1 \%$ Nonidet P- $40,0.5 \%$ sodium deoxycholate, and $1 \%$ sodium dodecyl sulfate; Beyotime, Shanghai, China) containing a 1:50 ratio of protease and phosphatase inhibitor cocktail (Roche, Basel, Switzerland). The protein samples were determined using a BCA kit (Solarbio Science \& Technology Co., Ltd., Beijing, China), resolved via sodium dodecyl sulfate-polyacrylamide gel electrophoresis (SDS-PAGE), and transferred to polyvinylidene difluoride (Millipore, USA) membranes. The primary antibodies against the following targets were used: anti-lba1 antibody (1:1000; Abcam, USA), anti-CD11b antibody (1:1000; Abcam, USA), anti-D3R antibody (1:2000; Abcam, USA), anti-GAPDH antibody (1:2000; Proteintech, USA), anti-phosphorylated ERK1/2 antibody (p-ERK1/2, 1:1000; CST, USA), anti- ERK1/2 antibody (ERK1/2, 1:1000; CST, USA), anti-phosphorylated P38 antibody (p-P38, 1:1000; CST, USA), anti-P38 antibody (1:1000; CST, USA), anti-phosphorylated JNK antibody (p-JNK, 1:1000; CST, USA), anti-JNK antibody (1:1000; CST, USA), anti-phosphorylated CREB antibody (p-CREB, 1:1000; CST, USA), anti-CREB antibody (1:1000; CST, USA), anti-phosphorylated Akt antibody (p-Akt, 1:1000; CST, USA), antiAkt antibody (1:1000; CST, USA). Proteins were visualized by incubation in ECL solution (Millipore, USA), and images were captured using a Fusion FX5 camera system. The scanned images were measured using ImageJ software (National Institute of Health, Bethesda, MA, USA). Specific bands were then quantified and normalized to the GAPDH loading control for each lane and each blot. 


\section{Cytokine assessment}

After NAc brain region and primary microglial and BV-2 cells were treated by LPS or IL-4 for $24 \mathrm{~h}$, the supernatants obtained from NAc tissue and cultured cells were collected. The concentrations of secreted TNF-a, IL-1 $\beta$, IL-6, IL-10 and Arginase- 1 were detected by enzyme-linked immunosorbent assay (ELISA) kits according to the manufacturer's instructions (Invitrogen, Thermo Fisher). Sample values were read from the standard curve. Each sample was assayed in duplicate.

\section{Flow cytometry}

The NAc brain tissue after virus administration were collected and placed in the $2 \mathrm{ml} \mathrm{RPMI}$ and disaggregated by grinders, and the collected cell suspension was filtered through a 70- $\mu \mathrm{m}$ strainer to prevent cell clumps and re-suspended in $200 \mathrm{~mL}$ PBS for detection. The BV-2 cells in the culture dish were digested using trypsin, and washed and re-suspended in PBS. All isolated cells from tissue and BV-2 cell were incubated with FITC-conjugated monoclonal mouse CD45 antibody (BioLegend, San Diego, USA), brilliant violet $421^{\mathrm{TM}}$-conjugated monoclonal mouse CD11 b antibody (BioLegend, San Diego, USA), PEconjugated monoclonal mouse CD86 antibody (BioLegend, San Diego, USA) and APC-conjugated monoclonal mouse CD206 antibody (BioLegend, San Diego, USA) for 30 min at $4^{\circ} \mathrm{C}$ in the dark. Finally, cells were fixed and measured using a Beckman Coulter flow cytometer.

\section{Immunofluorescence staining}

The primary microglial and BV-2 cells were seeded on coverslips and cultured in DMEM with $10 \%$ FBS for $24 \mathrm{~h}$. After LPS treatment, cells were washed twice in PBS and fixed in 4\% paraformaldehyde at room temperature for $30 \mathrm{~min}$, then permeabilized with Triton X-100 for $10 \mathrm{~min}$ and blocked with normal goat serum for $1 \mathrm{~h}$. The cells were stained by rabbit anti-mouse CD11b antibody (1:200; Abcam, USA) overnight at $4^{\circ} \mathrm{C}$ and then incubated with fluroscence-secondary antibody FITC (1:500; Proteintech, USA). Cells were treated with DAPI solution for $20 \mathrm{~min}$ at $37^{\circ} \mathrm{C}$. Images were acquired by a Fluorescence confocal microscope (Lecia SP8, Germany) and analyzed with ImageJ software system.

\section{Statistical analysis}

All data are presented as the mean \pm SEM. Data were analysed using IBM SPSS Statistics 20.0. No statistical methods were used to predetermine sample sizes, but our sample sizes are similar to those reported in previous publications $[25,29]$. Student's t-tests and one-way (treatment) analysis of variance (ANOVA) were performed as appropriate. After ANOVA was performed, the LSD post hoc test was used for multiple comparisons. P-values less than 0.05 were considered statistically significant.

\section{Results}

NAc injected with LPS-induced depressive-like phenotype is associated with the alterations of D3R and microglia 
Existing advances indicate a strong link between NAc inflammation and depression in response to social stress and saturated dietary fat [20,31], but there is no evidence that NAc inflammation may causally contribute to depressive illness. Thus, bilateral NAc injection of LPS-induced NAc inflammation mouse model was applied to address this problem in our present study. In results of a battery of depressionrelated behavioral tests after $24 \mathrm{~h}$ bilateral NAc injection of LPS, we observed a significant increase in immobility time in the FST ( $p=0.002$; Fig. $1 c)$ and TST ( $p=0.0001$; Fig. $1 \mathrm{~d})$, compared with those in the NAc saline-treated group. Likewise, results in the sucrose preference test showed that NAc injected with LPS inhibited preference for sucrose in mice relative to controls ( $p=0.0001 ; \mathrm{Fig}$. $1 \mathrm{e})$. These findings suggest that NAc inflammation induced by bilateral LPS injection would directly trigger depressive-like behaviors in rodents.

Considering that D3R and microglial activation play an important role in the depression from our and other published data $[13,23,25]$, we next characterize D3R and microglial expression in the bilateral NAc treated with LPS-induced depressive-like behaviors. As shown in Fig.1h, D3R mRNA in the NAc displayed a decline expression at $4 \mathrm{~h}(p=0.005), 12 \mathrm{~h}(p=0.0001)$ and $24 \mathrm{~h}(p=0.001)$ after NAc LPS treatment compared to control group, whereas Iba1-labelled microglia mRNA levels in the NAc were increased at 24 $\mathrm{h}(p=0.006)$ without at $4 \mathrm{~h}(p=0.844)$ and $12 \mathrm{~h}(p=0.224)$ after NAc LPS injection, indicating that D3R expression decline antecedent to microglia-labelled expression increase in the depressive-like behavioral model of bilateral NAc injection of LPS. Similarly, in results of protein levels of D3R (Fig.1f) and Iba1labelled micrglia in the NAc (Fig.1g), we found that D3R expression was decreased ( $p=0.005$, Fig. 1i) but Iba1-marked microglial expression ( $p=0.0001$, Fig.1j) was elevated after $24 \mathrm{~h}$ following bilateral NAc injected into LPS. In this context, D3R and microglia in the NAc were both implicated in bilateral NAc treated with LPS-induced depressive-like behaviors.

\section{Interaction between the D3R and microglia in the depressive-like behaviors induced by bilateral NAC injection of LPS}

We next used pharmacological intervention to further confirm the function of D3R and/or microglia in condition of bilateral NAc injected with LPS-induced depressive-like symptoms. A detailed experimental schematic is outlined in Fig. $2 a$, and behavioral results showed that pre-treatment with minocycline and PD128907 significantly decreased the extended immobility in the FST (one-way ANOVA, $F(3,19)=6.029$, $p=0.024$; one-way ANOVA, $\mathrm{F}(3,20)=12.115, p=0.002$, respectively; Fig. $2 \mathrm{~b}$ ) and TST (one-way ANOVA, $F(3,19)=6.039, p=0.024$; one-way ANOVA, $F(3,20)=6.261, p=0.021$, respectively; Fig. $2 c)$ after $24 \mathrm{~h}$ of NAc injected with LPS. Moreover, the decline in sucrose intake (Fig. 2d) in NAc LPS-exposed mice was augmented after treatment with either minocycline (one-way ANOVA, $\mathrm{F}(3,19)=9.444, p=0.006$ ) or PD128907 (one-way ANOVA, $F(3,20)=14.036, p=0.001$ ).

Next, we further explored the cross effects of D3R and microglia in the NAc on LPS challenge-triggered NAc inflammation and discovered that minocycline administration amplified the NAc LPS injectioninduced D3R expression decline in the NAc (one-way ANOVA, $F(3,19)=4.616, p=0.045$; Fig. 2e, g), as well as significantly alleviated NAc LPS injection-induced pro-inflammatory cytokines increase in the NAc 
(TNF-a: one-way ANOVA, $\mathrm{F}(3,20)=27.589, p=0.0001$; IL-1 $\beta$ : one-way ANOVA, $p=0.0001$; IL-6: one ANOVA, $\mathrm{F}(3,20)=32.399, p=0.0001$; Fig. 2i-k). In addition, PD128907 pre-treatment obviously inhibited the expression of Iba1-laballed microglia after NAc injection of LPS (one-way ANOVA, $F(3,19)=7.334$, $p=0.002$; Fig. 2f, h), and PD128907 treatment alleviated the elevated levels in TNF-a (one-way ANOVA, $F(3,20)=25.563, p=0.0001$; Fig. 2i) and IL-6 (one-way ANOVA, $F(3,20)=23.240, p=0.0001$; Fig. $2 \mathrm{k}$ ) but not in IL-1 $\beta$ level (one-way ANOVA, $F(3,20)=0.060, p=0.892$; Fig. $2 \mathrm{j}$ ) in the NAc. Together, these results indicate that interaction of D3R and microglia in the NAc, at least part, may be involved in NAc inflammation and thereby contribute to development of depressive-like behaviors induced by NAc inflammation.

\section{Down-regulation of D3R expression in the NAc induces depressive-like behaviors and microglia activation}

Despite of a strong correlation between D3R inhibition and microglia activation was implicated in depressive-like behaviors induced by inflammation, it remains to determine whether D3R and microglia have a causal relationship, which will be a significant for NAc inflammation-associated depression-like condition. First, the cultured microglial cells after down-regulated D3R expression (Fig. 3a, b) were used to examine a causal link between D3R and microglia. We observed that CD11b-labelled microglia from control BV-2 cells (Fig. 3d, e) displayed small soma and weak fluorescence intensity without LPS stimulation, but CD11b-labelled microglial cell body (Fig. 3f) and immunoreactivity were enhanced after LPS stimulation ( $p=0.045$, Fig. $3 c$ ). Remarkably, compared to BV-2 cells stimulated with LPS alone, D3R knockdown used by siRNA led to an evident higher intensity of CD11b staining ( $p=0.035$, Fig. $3 c)$ and larger CD11b-labelled microglial cell body (Fig. 3g) in BV-2 cells stimulated with LPS. Consistent with cultured BV-2 microglial cells, immunostaining results in the primary microglia cells obtained from D3RKO mice revealed that CD11b-labelled microglia had a larger cell body and more ramified process (Fig 3k) than the corresponding of WT primary microglia cells (Fig 3i) when subjected to LPS, and quantitative analysis of CD11b-labelled microglia from D3RKO mice after LPS stimulation also displayed marked increase in the fluorescence intensity (one-way ANOVA, $F(3,20)=26.308, p=0.0001$; Fig $3 \mathrm{I}$ ) and a substantial augment in cell areas (one-way ANOVA, $\mathrm{F}(3,20)=63.147, p=0.0001$; Fig $3 \mathrm{~m}$ ) when compared to those in WT mice. However, we did not observe any obvious morphological alterations in primary microglia cells obtained from both WT and D3RKO mice under normal circumstance (Fig 3h, j). These results demonstrated that D3R deficiency mediated microglial activation in inflammatory condition but not in normal baseline.

It is on this basis, we next further sought to a causal relationship of D3R inhibition and microglia activation in in vivo study. We selectively knocked out D3R expression in the NAc by administrating an AAV2/8 vector expressing a Drd3-targeting shRNA transcript or a control non-targeting vector to adult male mice to make a conditional knockout (CKO) of D3R in the NAc (Fig. 4a). After verifying the D3R mRNA expression in NAc of CKO mice was lower than that in its control mice ( $p=0.021$, Fig. $4 \mathrm{~d}$ ), all AAVinjected mice were then subjected to a series of depression-related behavioral tests as depicted in Fig.4c. Surprisingly, mice injected with AAV-shD3R spent more time immobile in the FST ( $p=0.005$; Fig. $4 f$ ) and TST ( $p=0.0001 ; \mathrm{Fig} .4 \mathrm{~g}$ ), as well as displayed anhedonia as assessed with the sucrose preference test ( $p=0.001$; Fig. $4 \mathrm{~h}$ ) when were comparable to AAV-control mice, which were consistent with the results 
obtained in global D3RKO mice demonstrating depressive-like behaviors. Afterwards, we performed a rescue experiment to confirm whether selectively restoring D3R expression in the NAc of adult global D3RKO mice would alleviate the depressive-like behaviors established by D3R mutation. Thus, adult D3RKO mice were injected AAV2/8-Drd3 (Fig. 4b) into bilateral NAc to over-express D3R in the NAc $(p=0.011$, Fig. 4e). Behavioral tests demonstrated that over-expression of D3R in the NAc were sufficient to rescue the observed depressive-like abnormalities from D3RKO control mice (FST: one-way ANOVA, $F(3,28)=5.646, p=0.004 ;$ TST: one-way ANOVA, $F(3,28)=6.378, p=0.002$; Sucrose preference test: one-way ANOVA, $F(3,28)=34.475, p=0.0001 ;$ Fig. $4 \mathrm{i}-\mathrm{k})$. These results indicate that D3R in the NAc exerts an important role in the initiation and development of depression-like behaviors. Importantly, we finally measured the microglia changes in the NAc in following knocked out D3R expression by flow cytometry. The results demonstrated that microglia in the NAc (Fig. 5a, b), presented as CD $45^{\text {int }} \mathrm{CD} 11 \mathrm{~b}^{+}$cells, increased significantly in adult D3R CKO mice, compared to control groups ( $p=0.013$, Fig. $5 \mathrm{~d}$ ), further corroborating the presence of microglia proliferation after D3R expression inhibition. Taken together, our findings provide a notion that down-regulated D3R expression in the NAc would promote microglia activation and consequent neuroinflammation and depressive-like behavior.

\section{D3R inhibition in the NAc promotes microglial polarization to the M1 phenotype}

Taking into account two main polarization states of activated microglia in response to stimulus, we sought to test whether inhibition of D3R could potentially influence microglial polarization in CKO mice. CD86 was used as the marker of M1 polarization, while CD206 was employed as the marker of M2 polarization. As shown in Fig. $5 a, b, \mathrm{CD}_{8} 6^{+} \mathrm{M} 1$ microglia in the NAc was markedly up-regulated in the D3R CKO mice compared with that of control mice ( $p=0.001$, Fig.5e). However, there were no statistical changes of $\mathrm{CD}_{206}{ }^{+} \mathrm{M} 2$ microglia in the NAc between CKO and control mice ( $p=0.710$, Fig.5f). These results indicated that suppression of D3R in the NAc converts microglia to the M1 phenotype but not the M2 phenotype, which probably results in microglia activation and NAc inflammation.

Next, cultured microglial cells were used to further verify the mechanism of D3R in microglia activation. In cultured BV-2 cells, a pro-inflammatory stimuli LPS was applied to induce M1 microglia phenotype but an anti-inflammatory cytokine IL-4 was used to trigger M2 microglia phenotype. We detected that CD86 expression (Fig.5g) was higher after LPS stimulation than control groups ( $p=0.0001$, Fig.5i). Importantly, compared with the LPS treatment alone, suppression of D3R induced by siRNA exacerbated CD86 expression in cultured BV-2 cells after LPS exposure ( $p=0.0001$, Fig.5i). In results of cytokine levels produced by LPS (Supplementary Fig.1a-e), the pro-inflammatory cytokines expression of TNF-a $(p=0.004)$ \IL-1 $\beta(p=0.016)$ and IL-6 $(p=0.0001)$ were increased significantly compared to control group, and inhibition in D3R aggravated pro-inflammatory cytokine production (TNF-a: $p=0.041$; IL-1 $\beta$ : $p=0.045$; IL-6: $p=0.014)$ stimulated by LPS compared to that of LPS treatment alone; While anti-inflammatory molecules IL-10 and arginase-1 expression did not show any differences in the cultured BV-2 microglial cells among different treatment groups. In accordance with these results, cultured primary microglial cells (Supplementary Fig.1f-j) from D3RKO mice stimulated with LPS showed a significant increase in proinflammatory cytokines (TNF-a: $p=0.006$; IL-1 $\beta$ : $p=0.0001$; IL-6: $p=0.0001$ ) but did not affect anti- 
inflammatory molecules, when compared to WT mice following LPS challenge. These studies sufficiently demonstrated that inhibition of D3R triggers M1 phenotype of activated microglia. In addition, The CD206 expression (Fig.5h) was significantly elevated in the cultured BV-2 cells following IL-4 treatment ( $p=0.0001$, Fig.5j), but inhibition of D3R expression did not change IL-4 stimulation-induced CD206 expression in BV-2 microglial cells ( $p=0.689$, Fig.5j). Also, we observed an obvious decline in proinflammatory cytokines (TNF-a: $p=0.0001$; IL-1 $\beta$ : $p=0.0001$; IL-6: $p=0.001$ ) and elevation in antiinflammatory molecules (IL-10: $p=0.0001$; arginase-1: $p=0.0001$ ) in cultured BV-2 cells subjected to IL-4 treatment, but down-regulation of D3R did not affect inflammatory cytokines production following IL-4 stimulus compared to IL-4 treatment alone (Supplementary Fig. 2a-e). These results showed that downregulation in D3R expression did not induce M2 phenotype of activated microglia.

\section{Effects of Akt signaling pathway on microglia activation induced by D3R inhibition}

It has been reported that D3R plays an important role in neurogenesis and neuropsychiatric disorders mainly through MAPK, AKt and CREB signaling pathways [21, 32-34]. Thus we next screen a series of related signaling pathways that may participate in D3R inhibition-induced microglia activation in cultured BV-2 cell line. Western blot analysis (Fig.6f) showed that LPS stimulation resulted in expression of $p$-JNK $(p=0.031$, Fig.6c) enhanced without affecting JNK expression ( $p=0.586$, Fig. $6 c)$ and activation ( $p$ JNK/JNK; $p=0.562$, Fig.6c) in BV-2 cells compared to control group. Combination effects of D3R inhibition and LPS in the BV-2 cells aggravated JNK activation ( $p=0.042$, Fig.6c) and $p$-JNK protein expression ( $p=0.039$, Fig.6c) but not JNK expression ( $p=0.282$, Fig.6c), compared with LPS treatment alone. Also, co-effects of D3R inhibition and LPS promoted Akt activation ( $p=0.010$, Fig.6e) and p-Akt expression ( $p=0.003$, Fig.6e) but not Akt expression ( $p=0.301$, Fig.6e) in the BV-2 cells compared to LPS treatment alone, but we did not detect any changes in expression of $\mathrm{p}$-Akt ( $p=7.000$, Fig.6e) and Akt ( $p=0.270$, Fig.6e) and activation of Akt ( $p=0.092$, Fig.6e) stimulated by LPS compared to these in control group. However, the expression and activation of P38 pathway in BV-2 cells had no difference after LPS or siRNA treatment (Fig.6b); and EKR1/2 (Fig.6a) and CREB (Fig.6d) pathway expression and activation in BV-2 cells presented upward tendency but no statistical difference among LPS and siDrd3 treatment. These results suggest that JNK and Akt signaling pathways seem to be implicated in D3R inhibitioninduced microglia activation. To further confirm which signaling pathway influences the D3R inhibitiontriggered microglia activation in BV-2 cells, $\mathrm{CD} 86^{+} \mathrm{M} 1$ microglia expression was measured after pretreatment with different doses of JNK (SP600125) and Akt (MK2206) pathway inhibitors in BV-2 cells stimulated by LPS. The dosage selection and treatment duration were chose based on preliminary experiments to achieve a balance between enhancement of drugs activities and minimal potential side effects. As indicated in Fig. $6 \mathrm{~g}$, $\mathrm{h}$ and Supplementary Fig.2a, we found that suppression of D3R expression induced by siDrd3 promoted CD86 expression compared to control group after LPS treatment. However, in the condition of LPS stimulation, $20 \mathrm{uM}(p=0.0001)$ and $40 \mathrm{uM}(p=0.0001)$ MK2206 treatment dose-dependently attenuated CD86 expression stimulated with siDrd3 in BV-2 cells when compared these to corresponding control group, whereas SP600125 pre-treatment at $80 \mathrm{uM}$ and $160 \mathrm{uM}$ did not show any statistical difference in CD86 expression after stimulated with siDrd3 in BV-2 cells, 
although 160 uM SP600125 could alleviate CD86 expression increase in BV-2 cells after LPS and siControl treatment $(p=0.046)$. These findings reveal that $\mathrm{D} 3 \mathrm{R}$ inhibition induces microglial polarization to the M1 phenotype mainly through Akt signalling pathway in response to inflammation.

\section{Discussion}

Our study provides direct evidence showing that NAc inflammation leaded to depressive-like disorders, and observing the interaction of D3R and microglia activation involved in NAc inflammation-induced depressive-like phenotype. The results obtained from selective knock-down and re-expression of D3R in the NAc showed a crucial role of D3R in the initiation and development of depressive-like behaviors. Importantly, D3R in the NAc mediated microglial polarization to M1 phenotype mainly through Akt signaling pathway that possibly contributed to NAc inflammation and then depressive-like behaviors.

As an intricate neuroanatomical connectivity pattern, NAc alterations presented in molecular expression, synaptic plasticity and structural plasticity have been reported to be associated with anhedonia and motivational deficits that are core symptoms of depression [35,36]. Despite a few recent studies pointing out that inflammatory responses in the NAc are strongly correlated with the development of depressive illness in animal models induced by stress, excessive intake of high-fat/sucrose diets and peripheral LPS challenge [20,21,31], there has been no direct evidence on the causal relationship between inflammation occurring in the NAc and the establishment of depressive-like behaviors. To address this issue, a model of LPS injection into the NAc to mimic NAc inflammation was employed in our current study. As expected, we observed that direct intra-NAc injection of LPS not only facilitated an increase in NAc microglia activation as well as pro-inflammatory cytokines, such as TNF- $a, \mathrm{IL}-1 \beta$ and IL-6, but also triggered a series of depressive-like behaviors; while that behavioral despair was alleviated by inhibition of microglia activation. Consistently, LPS challenge, as an immune trigger to elicit immune responses, could active microglia in the brain along with pro-inflammatory cytokines production and then promotes neuroinflammation $[11,12]$. Taken together, such present findings echo and extend previous studies showing that inflammation occurring in the NAc was implicated in depression and further provide direct support for NAc inflammation-induced depressive-like behaviors.

Specifically, in rodent model of NAc LPS-induced inflammation and depressive-like behaviors, D3R mRNA and protein expression we observed markedly decreased in the NAc, and we discovered the decline in D3R mRNA in the NAc anterior to microglia activation. However, microinjection of the D3R activator PD128907 into the NAc attenuated NAc LPS challenge-triggered production of the proinflammatory cytokines TNF-a and IL-6 and depressive-like behaviors. That intriguing result for the role of D3R in the NAc in inflammation relevant depression, coupled with D3R as a potential candidate target for antidepressants, arouses us to further investigate mechanisms for D3R expressed in the NAc in the depressive-like behaviors. Surprisingly, we found that selectively knock-down D3R expression in the NAc in adult mice sufficiency produced depressive-like behaviors. Also selectively restoring D3R expression in the NAc of adult global D3RKO mice would alleviate the depressive-like behaviors established by D3R mutation. Indeed, previous studies have reported that D3R specially located at the limbic projection areas 
associated with motivational and emotional functions and has been implicated in the pathophysiology of depression $[23,24,37]$. In this context, our present results obtained from global KO and CKO of D3R and the rescuing experiment strongly support the notion that D3R expressed in the NAc plays a pivotal role in the development and progress of depressive-like illness and highlight the significant of D3R in future pharmacological target in the depression.

Microglia are the intrinsic immune cells of the brain and express a variety of neurotransmitter receptors, including glutamate, $y$-aminobutyric acid (GABA), norepinephrine, cannabinoid and acetylcholine receptors [38,39]. In this regard, our published in vivo studies have demonstrated that D3R was distributed in microglial cells in the NAc in mouse brain parenchyma under normal physiological conditions [25], suggesting a possible role of D3R in the microglia. Importantly, we observed from our present study that inhibition in D3R altered microglial morphology and conversed to activated state when cultured BV-2 and primary microglia cells are stimulated with LPS; similarly, down-regulated D3R expression in the NAc in adult mice promoted microglial proliferation. Thus, our present findings provide direct experimental evidence that D3R expressed in the NAc exerts causal effects on microglia activation and partially subsequent the development of depression-like behaviors. Nevertheless, given previous studies have revealed that neurotransmitter receptors expressed in microglia help to mediate the bidirectional communication between neurons and microglia, mainly showing neurotransmitters released from aberrant neuronal activities could influence microglia action [39-42]. Therefore, during the decrease of D3R expression in the NAc affects microglia state, we could not rule out the possibility the crosstalk of neuron and microglia after D3R expression down-regulated that thereby alter microglia state, not merely the direct role of D3R expressed on microglia mediates itself activation. Furthermore, we previously consider that microglia activation would be the compensatory changes throughout development in global D3RKO mice, whereas selectively knock-down D3R in the NAc is done in adulthood, so any compensatory changes will likely be fewer and mechanistically different.

Previous studies have shown that activated microglia can be transformed into two main polarizations: the classically activated M1 (pro-inflammatory) and alternatively activated M2 (anti-inflammatory) phenotypes. Activated M1 microglia are usually induced by pro-inflammatory stimuli (such as LPS) and characterized by the increased release of pro-inflammatory cytokines (e.g. TNF- $a$, IL-1 $\beta$ and IL-6). By contrast, activated M2 microglia is mainly induced by IL-4 and/or IL-13, which lead to produce antiinflammatory cytokines such as IL-10 and are involved in phagocytosis of cell debris, neuron protection and tissue repair [43-45]. In our present in vivo result, we found that down-regulation of D3R in the NAC promoted activated microglia to M1 phenotype but failed to M2 phenotype. Similar to these results, in vitro cultured cells showed that BV-2 microglia not only presented M1 phenotype induction as evidenced by the pronounced expression characteristic markers CD86 and pro-inflammatory cytokines (TNF-a, IL-1 $\beta$ and IL-6) after LPS exposure, but also inhibition of D3R in the cultured BV-2 cells exacerbated M1 phenotype characteristic markers expression when exposed to LPS. However, inhibition of D3R did not affect the augment levels of CD206, Arginase- 1 and IL-10 in the IL-4 treatment-induced M2 phenotype of BV-2 microglia, compared to IL-4 treatment alone. These in vivo and in vitro studies suggest that the decrease of D3R expression in the NAc mainly converses activated microglia to M1 phenotype and 
promotes pro-inflammatory cytokines that seem to result in NAc inflammation and depressive-like abnormalities. Finally, we measured several D3R relevant signaling pathways in the BV-2 cultured cells to determine the exact signaling pathway involved in D3R expression decrease-induced activated M1 microglia. In the results from BV-2 cells after combination treatment of LPS and inhibition in D3R, we discovered that JNK and Akt signaling pathways possibly associated with D3R inhibition-induced microglia activation. After verify the function of above two signalling pathways used by pre-treatment with their inhibitors, we determined that D3R inhibition induced microglial polarization to the M1 phenotype mainly through Akt signalling pathway in response to inflammation. Indeed, previous studies have reported that the stimulation of D3R transiently activates the Akt signaling pathway in dorsal striatum and NAc [46]. And D3R-mediated Akt signaling pathway plays a crucial role in the expression of nicotine-induced structural plasticity in mesencephalic dopaminergic neurons [47].

\section{Conclusions}

Overall, our study first observes a strong correlation of D3R and microglia activation in the NAC inflammation-induced depressive-like behaviors. On that basis, we further discover that down-regulation in D3R in the NAc elicits the establishment of depressive-like behaviors, as well as promotes activated microglia to M1 phenotype that possibly through Akt signaling pathway, which provides a causal evidence between D3R and microglia activation in the NAc, and would help develop novel targeted therapeutics for D3R involved in depression-like phenotype.

\section{Abbreviations}

D3R: dopamine D3 receptor; IL-1ß: interleukin-1 beta; IL-6: interleukin-6; TNF-a: tumour necrosis factor-a; NAc: nucleus accumbens; PET: positron emission tomography; TSPO: translocator protein 18 kD; SSRIs: serotonin reuptake inhibitors; D3RKO: dopamine D3 receptor-knockout; LPS: Lipopolysaccharide; DMEM: Dulbecco's modified Eagle's medium; FBS: fetal bovine serum; siRNA: small interfering RNA; PMM: Primary murine microglia

\section{Declarations}

\section{Acknowledgements}

The authors want to thank Dr. Xu for sharing the D3RKO mice and all the staff of the Key Laboratory of the Health Ministry for Medicine of Xi'an Jiaotong University for supporting the animal behavioral experiments.

\section{Funding}

This work was supported by the Natural Science Foundation of China (grant numbers 81901370 and 81871537), and China Postdoctoral Science Foundation (grand number 2019M653665) 


\section{Availability of data and materials}

Data and materials are available to interested parties on reasonable request

\section{Authors' contributions}

YJC and JW conceptualized and designed the study. JW conducted all the experiments and was involved in the analysis and interpretation of the data, as well as the writing of the manuscript. TZ and RW assisted in the generation of all data and analysed all figures. SML, ND and LZ were responsible for raising animals, sample collection and qRT-PCR. TC conceived the study and participated in the revision of the manuscript. All the authors have read and approved the manuscript.

\section{Ethics approval and consent to participate}

All procedures were performed in accordance with the guidelines of the Institutional Animal Care and Use Committee of Xi'an Jiaotong University and were approved by the Animal Ethics Committee of Xi'an Jiaotong University.

\section{Consent for publication}

All authors consent to publication.

\section{Competing interests}

The authors declare that they have no competing interests

\section{Publisher's note}

Springer Nature remains neutral with regard to jurisdictional claims in published maps and institutional affiliations.

\section{References}

1. Lopez AD, Murray CC. The global burden of disease. 1990-2020. Nat Med. 1998;4(11):1241-3.

2. Mathers CD, Loncar D. Projections of global mortality and burden of disease from 2002 to 2030. PLoS Med. 2006;3(11):e442.

3. Malhi GS, Mann JJ. Depression. Lancet. 2018;392(10161):2299-2312.

4. Beurel E, Toups M, Nemeroff CB. The Bidirectional Relationship of Depression and Inflammation: Double Trouble. Neuron. 2020;107(2):234-256.

5. Rush AJ, Trivedi MH, Wisniewski SR, Nierenberg AA, Stewart JW, Warden D, et al. Acute and longerterm outcomes in depressed outpatients requiring one or several treatment steps: a STAR*D report. Am J Psychiatry. 2006;163(11):1905-17. 
6. Kettenmann H, Kirchhoff F, Verkhratsky A. Microglia: new roles for the synaptic stripper. Neuron. 2013;77(1):10-8.

7. Kettenmann H, Hanisch UK, Noda M, Verkhratsky A. Physiology of microglia. Physiol Rev. 2011;91(2):461-553.

8. Torres-Platas SG, Cruceanu C, Chen GG, Turecki G, Mechawar N. Evidence for increased microglial priming and macrophage recruitment in the dorsal anterior cingulate white matter of depressed suicides. Brain Behav Immun. 2014;42:50-9.

9. Rupprecht R, Papadopoulos V, Rammes G, Baghai TC, Fan J, Akula N, et al. Translocator protein (18 kDa) (TSPO) as a therapeutic target for neurological and psychiatric disorders. Nat Rev Drug Discov. 2010;9(12):971-88.

10. Banati RB. Visualising microglial activation in vivo. Glia. 2002;40(2):206-17.

11. Hoogland IC, Houbolt C, van Westerloo DJ, van Gool WA, van de Beek D. Systemic inflammation and microglial activation: systematic review of animal experiments. J Neuroinflammation. 2015;12:114.

12. Guan YF, Huang GB, Xu MD, Gao F, Lin S, Huang J, et al. Anti-depression effects of ketogenic diet are mediated via the restoration of microglial activation and neuronal excitability in the lateral habenula. Brain Behav Immun. 2020;88:748-762.

13. Jia X, Gao Z, Hu H. Microglia in depression: current perspectives. Sci China Life Sci. 2020.

14. Lehmann ML, Weigel TK, Poffenberger CN, Herkenham M. The Behavioral Sequelae of Social Defeat Require Microglia and Are Driven by Oxidative Stress in Mice. J Neurosci. 2019;39(28):5594-5605.

15. Zhang C, Zhang YP, Li YY, Liu BP, Wang HY, Li KW, et al. Minocycline ameliorates depressive behaviors and neuro-immune dysfunction induced by chronic unpredictable mild stress in the rat. Behav Brain Res, 2019;356:348-357.

16. Russo SJ, Nestler EJ. The brain reward circuitry in mood disorders. Nat Rev Neurosci. 2013;14(9):609-25.

17. Floresco SB. The nucleus accumbens: an interface between cognition, emotion, and action. Annu Rev Psychol. 2015;66:25-52.

18. Francis TC, Lobo MK. Emerging Role for Nucleus Accumbens Medium Spiny Neuron Subtypes in Depression. Biol Psychiatry. 2017;81(8):645-653.

19. Farooq RK, Isingrini E, Tanti A, Le Guisquet AM, Arlicot N, Minier F, et al. Is unpredictable chronic mild stress (UCMS) a reliable model to study depression-induced neuroinflammation. Behav Brain Res, 2012;231(1):130-7.

20. Décarie-Spain L, Sharma S, Hryhorczuk C, Issa-Garcia V, Barker PA, Arbour N, et al. Nucleus accumbens inflammation mediates anxiodepressive behavior and compulsive sucrose seeking elicited by saturated dietary fat. Mol Metab. 2018;10:1-13.

21. Wang J, Jia Y, Li G, Wang B, Zhou T, Zhu L, et al. The Dopamine Receptor D3 Regulates Lipopolysaccharide-Induced Depressive-Like Behavior in Mice. Int J Neuropsychopharmacol. 2018;21(5):448-460. 
22. Harrison NA, Brydon L, Walker C, Gray MA, Steptoe A, Critchley HD. Inflammation causes mood changes through alterations in subgenual cingulate activity and mesolimbic connectivity. Biol Psychiatry. 2009;66(5):407-14.

23. Leggio GM, Bucolo C, Platania CB, Salomone S, Drago F. Current drug treatments targeting dopamine D3 receptor. Pharmacol Ther. 2016;165:164-77.

24. Moraga-Amaro R, Gonzalez H, Pacheco R, Stehberg J. Dopamine receptor D3 deficiency results in chronic depression and anxiety. Behav Brain Res. 2014;274:186-93.

25. Wang J, Lai S, Li G, Zhou T, Wang B, Cao F, et al. Microglial activation contributes to depressive-like behavior in dopamine D3 receptor knockout mice. Brain Behav Immun. 2020;83:226-238.

26. Tsuchimine S, Yasui-Furukori N, Nakagami T, Sato Y, Kaneko S. DRD3, but not BDNF, genotype affects treatment response to paroxetine in major depressive disorder: a preliminary study. J Clin Psychopharmacol. 2012;32(5):724-6.

27. Newman AH, Blaylock BL, Nader MA, Bergman J, Sibley DR, Skolnick P. Medication discovery for addiction: translating the dopamine D3 receptor hypothesis. Biochem Pharmacol. 2012;84(7):882-90.

28. Lian H, Roy E, Zheng H. Protocol for Primary Microglial Culture Preparation. Bio Protoc. 2016;6(21).

29. Lawson MA, McCusker RH, Kelley KW. Interleukin-1 beta converting enzyme is necessary for development of depression-like behavior following intracerebroventricular administration of lipopolysaccharide to mice. J Neuroinflammation. 2013;10:54.

30. Livak KJ, Schmittgen TD. Analysis of relative gene expression data using real-time quantitative PCR and the 2(-Delta Delta C(T)) Method. Methods. 2001;25(4):402-8.

31. Menard C, Pfau ML, Hodes GE, Kana V, Wang VX, Bouchard S, et al. Social stress induces neurovascular pathology promoting depression. Nat Neurosci. 2017;20(12):1752-1760.

32. Reyes-Resina I, Awad AH, Del SA, Sánchez-Naves J, Lillo J, Jiménez J, et al. Expression of Melatonin and Dopamine D3 Receptor Heteromers in Eye Ciliary Body Epithelial Cells and Negative Correlation with Ocular Hypertension. Cells. 2020;9(1).

33. Lao CL, Lu CS, Chen JC. Dopamine D3 receptor activation promotes neural stem/progenitor cell proliferation through AKT and ERK1/2 pathways and expands type-B and $-\mathrm{C}$ cells in adult subventricular zone. Glia. 2013;61(4):475-89.

34. Collo G, Bono F, Cavalleri L, Plebani L, Pich EM, Millan MJ, et al. Pre-synaptic dopamine D(3) receptor mediates cocaine-induced structural plasticity in mesencephalic dopaminergic neurons via ERK and Akt pathways. J Neurochem. 2012;120(5):765-78.

35. Krishnan V, Nestler EJ. The molecular neurobiology of depression. Nature. 2008;455(7215):894-902.

36. Jiang C, Lin WJ, Sadahiro M, Labonté B, Menard C, Pfau ML, et al. VGF function in depression and antidepressant efficacy. Mol Psychiatry. 2018;23(7):1632-1642.

37. Lévesque D, Diaz J, Pilon C, Martres MP, Giros B, Souil E, et al. Identification, characterization, and localization of the dopamine D3 receptor in rat brain using 7-[3H]hydroxy-N,N-di-n-propyl-2aminotetralin. Proc Natl Acad Sci U S A. 1992;89(17):8155-9. 
38. Pocock JM, Kettenmann H. Neurotransmitter receptors on microglia. Trends Neurosci. 2007;30(10):527-35.

39. Liu H, Leak RK, Hu X. Neurotransmitter receptors on microglia. Stroke Vasc Neurol. 2016;1(2):52-58.

40. Fontainhas AM, Wang M, Liang KJ, Chen S, Mettu P, Damani M, et al. Microglial morphology and dynamic behavior is regulated by ionotropic glutamatergic and GABAergic neurotransmission. PLoS One. 2011;6(1):e15973.

41. Dissing-Olesen L, LeDue JM, Rungta RL, Hefendehl JK, Choi HB, MacVicar BA. Activation of neuronal NMDA receptors triggers transient ATP-mediated microglial process outgrowth. J Neurosci. 2014;34(32):10511-27.

42. Basso AM, Bratcher NA, Harris RR, Jarvis MF, Decker MW, Rueter LE. Behavioral profile of P2X7 receptor knockout mice in animal models of depression and anxiety: relevance for neuropsychiatric disorders. Behav Brain Res. 2009;198(1):83-90.

43. Boche D, Perry VH, Nicoll JA. Review: activation patterns of microglia and their identification in the human brain. Neuropathol Appl Neurobiol. 2013;39(1):3-18.

44. Saijo K, Glass CK. Microglial cell origin and phenotypes in health and disease. Nat Rev Immunol. 2011;11(11):775-87.

45. Tang Y, Le W. Differential Roles of M1 and M2 Microglia in Neurodegenerative Diseases. Mol Neurobiol. 2016;53(2):1181-1194.

46. Salles MJ, Hervé D, Rivet JM, Longueville S, Millan MJ, Girault JA, et al. Transient and rapid activation of Akt/GSK-3 $\beta$ and $m$ TORC1 signaling by D3 dopamine receptor stimulation in dorsal striatum and nucleus accumbens. J Neurochem. 2013;125(4):532-44.

47. Collo G, Bono F, Cavalleri L, Plebani L, Mitola S, Pich EM, et al. Nicotine-induced structural plasticity in mesencephalic dopaminergic neurons is mediated by dopamine D3 receptors and Akt-mTORC1 signaling. Mol Pharmacol. 2013;83(6):1176-89.

\section{Figures}


A

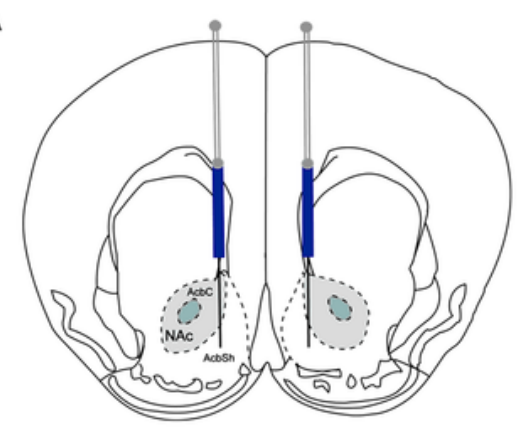

B

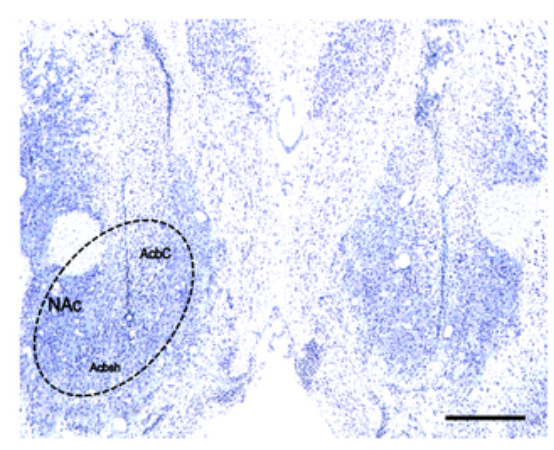

C

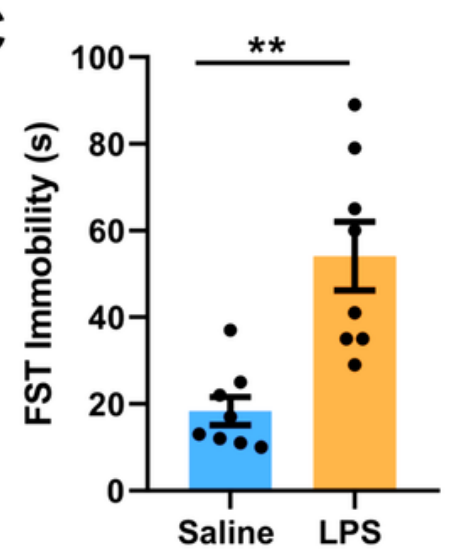

D

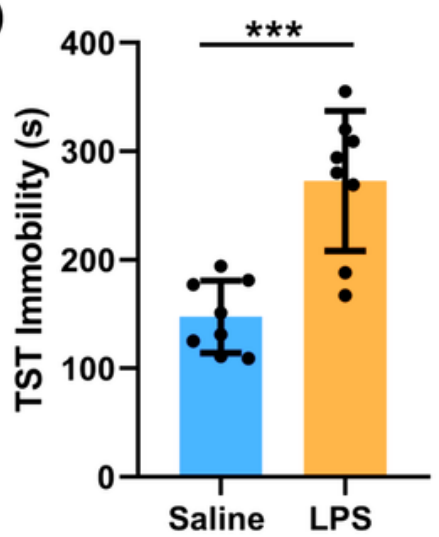

$\mathrm{E}$

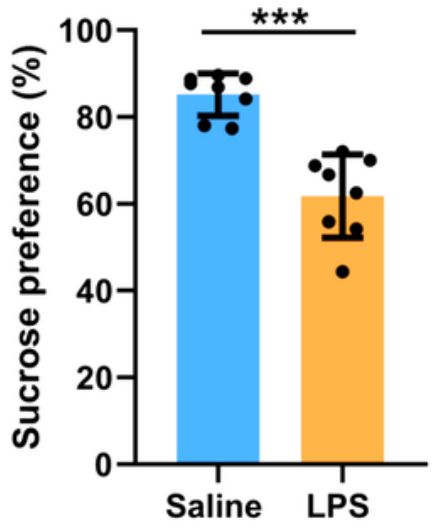

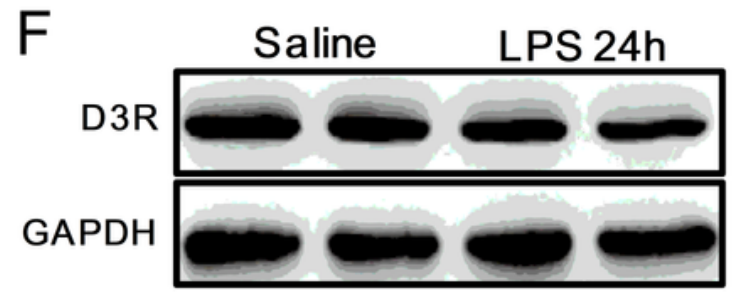

G

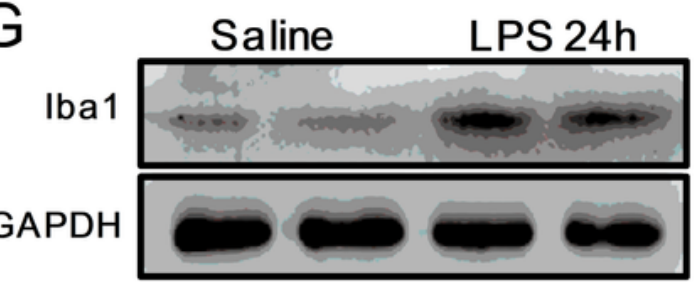

$\mathrm{H}$
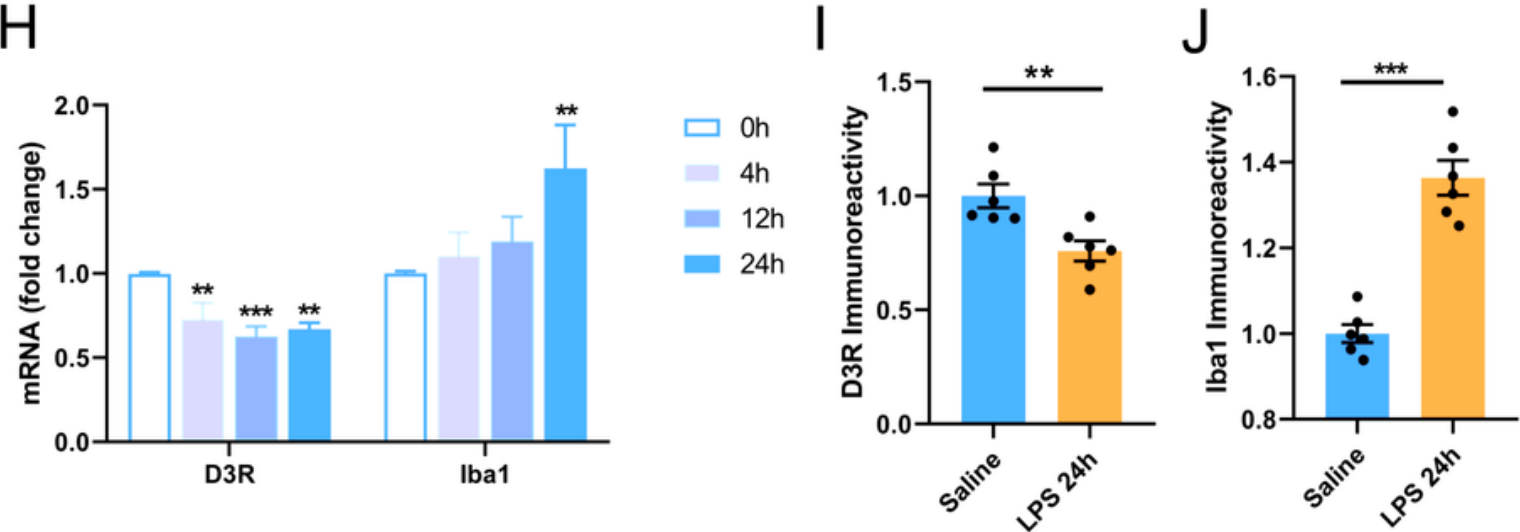

Figure 1

NAc injected with LPS-induced depressive-like phenotype is associated with the alterations of D3R and microglia. (a) Schematic showing bilateral administration of saline or LPS (100 ng) into the NAc. (b) An image indicating the actual injection site in the bilateral NAc staining by the Nissl method (scale bar 500 $\mu \mathrm{m}$ ). (c) The immobility time in the forced swim test (FST) was greater at $24 \mathrm{~h}$ after bilateral NAc LPS administration than in the saline groups ( $n=8 /$ group). (d) Similarly, a significant increase in immobility 
time in the tail suspension test (TST) $24 \mathrm{~h}$ after bilateral NAc LPS injection compared with those in the NAc saline-treated group ( $n=8 /$ group). (e) In the sucrose preference test, compared with the normal saline group, LPS-treated mice displayed a reduction in sucrose preference during $24 \mathrm{~h}$ period following NAc injection ( $n=8 /$ group). (f) Western blot showed that D3R protein expression in the NAc was decreased after $24 \mathrm{~h}$ of LPS treatment when compared to saline group. (g) Compared to saline group, intra-NAc administration of LPS elevated the Iba1-labelled microglia protein levels in the NAc at $24 \mathrm{~h}$ following injection of LPS. (h) Compared to control group ( $0 \mathrm{~h})$, D3R mRNA in the NAc displayed a decline expression at $4 \mathrm{~h}, 12 \mathrm{~h}$ and $24 \mathrm{~h}$, while Iba1-labelled microglia mRNA levels in the NAc were increased at $24 \mathrm{~h}$ without at $4 \mathrm{~h}$ and $12 \mathrm{~h}$ after NAc LPS injection ( $\mathrm{n}=6 /$ group). $(\mathrm{i}, \mathrm{j})$ Quantitative western blot analysis of D3R and Iba1-labelled microglia protein levels in the NAc $24 \mathrm{~h}$ following saline or LPS administration ( $n=6 /$ group). The data are presented as the mean \pm SEM. $* * p \otimes 0.01, * \star * p \otimes 0.001$. 

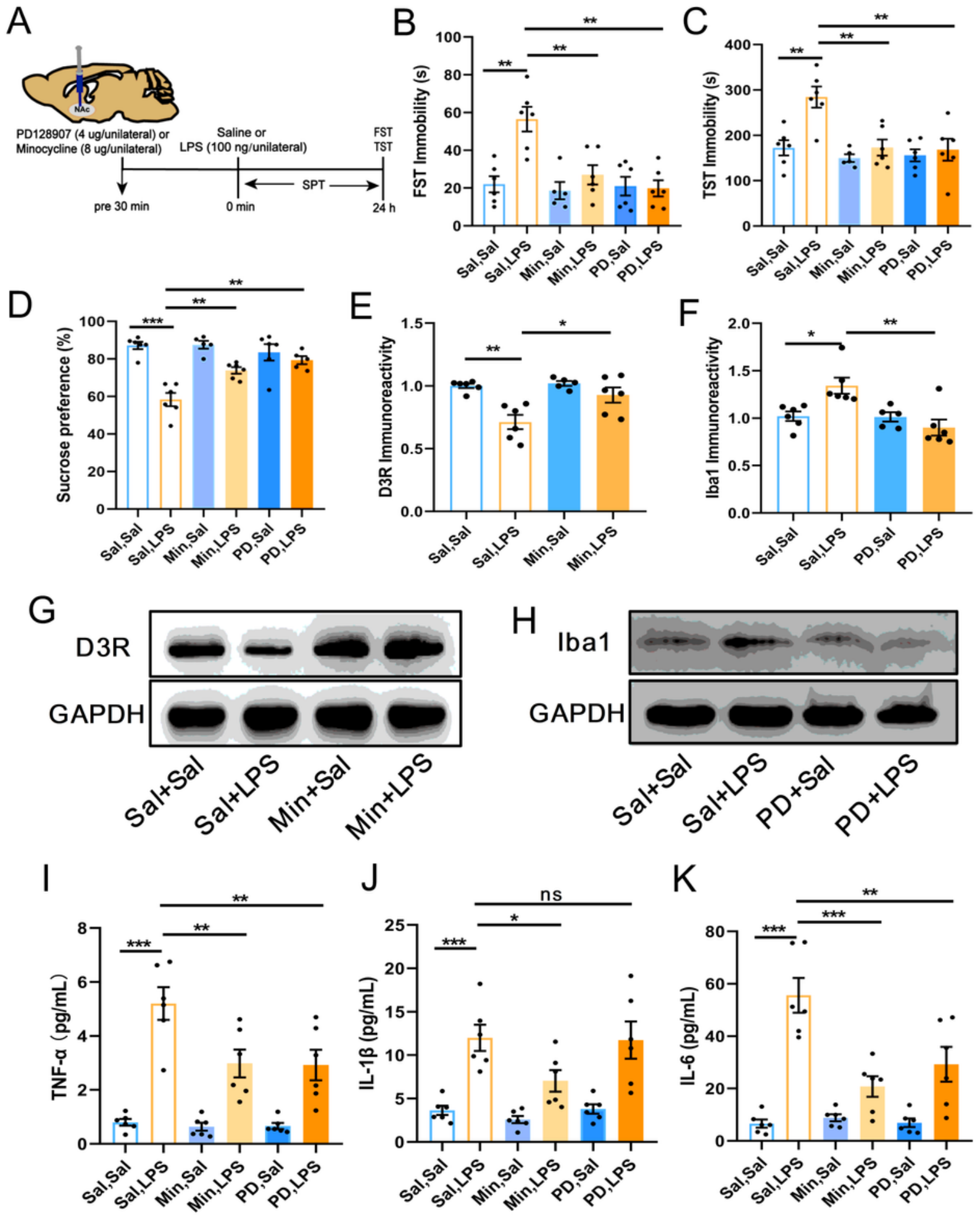

\section{Figure 2}

Interaction between the D3R and microglia in the depressive-like behaviors induced by bilateral NAc injection of LPS. (a) Illustration of the experimental design for minocycline and PD128907 treatments. (bd) Pre-treatment with minocycline and PD128907 significantly decreased NAc LPS treatment-induced the extended immobility in the FST and TST, and the decline in sucrose intake in NAc LPS-exposed mice was augmented after treatment with either minocycline or PD128907 ( $n=5-6 /$ group). (e-f) Quantitative western 
blot analysis of D3R and Iba1-labelled microglia relative densities in different experimental groups in the NAc after respective minocycline and PD128907 treatments ( $n=5-6 / g r o u p) .(g)$ Minocycline treatment increased NAc LPS injection-induced D3R expression decline in the NAc. (h) PD128907 treatment decreased the elevated expression of microglial Iba1 induced by NAc LPS injection in the NAc. (i-k) minocycline administration significantly alleviated NAc LPS injection-induced pro-inflammatory cytokines TNF- $\alpha$, IL-1 $\beta$ and IL- 6 increase in the NAc, PD128907 treatment alleviated the elevate levels in TNF- $\alpha$ and IL- 6 but not in IL-1 $\beta$ level ( $n=6 /$ group). The data are presented as the mean $\pm S E M$. One-way ANOVA followed by LSD's multiple comparisons post hoc test were used to assess significant differences. ${ }^{*} \mathrm{p} \rrbracket$ $0.05, * \star p \otimes 0.01, * \star \star p \otimes 0.001$. 

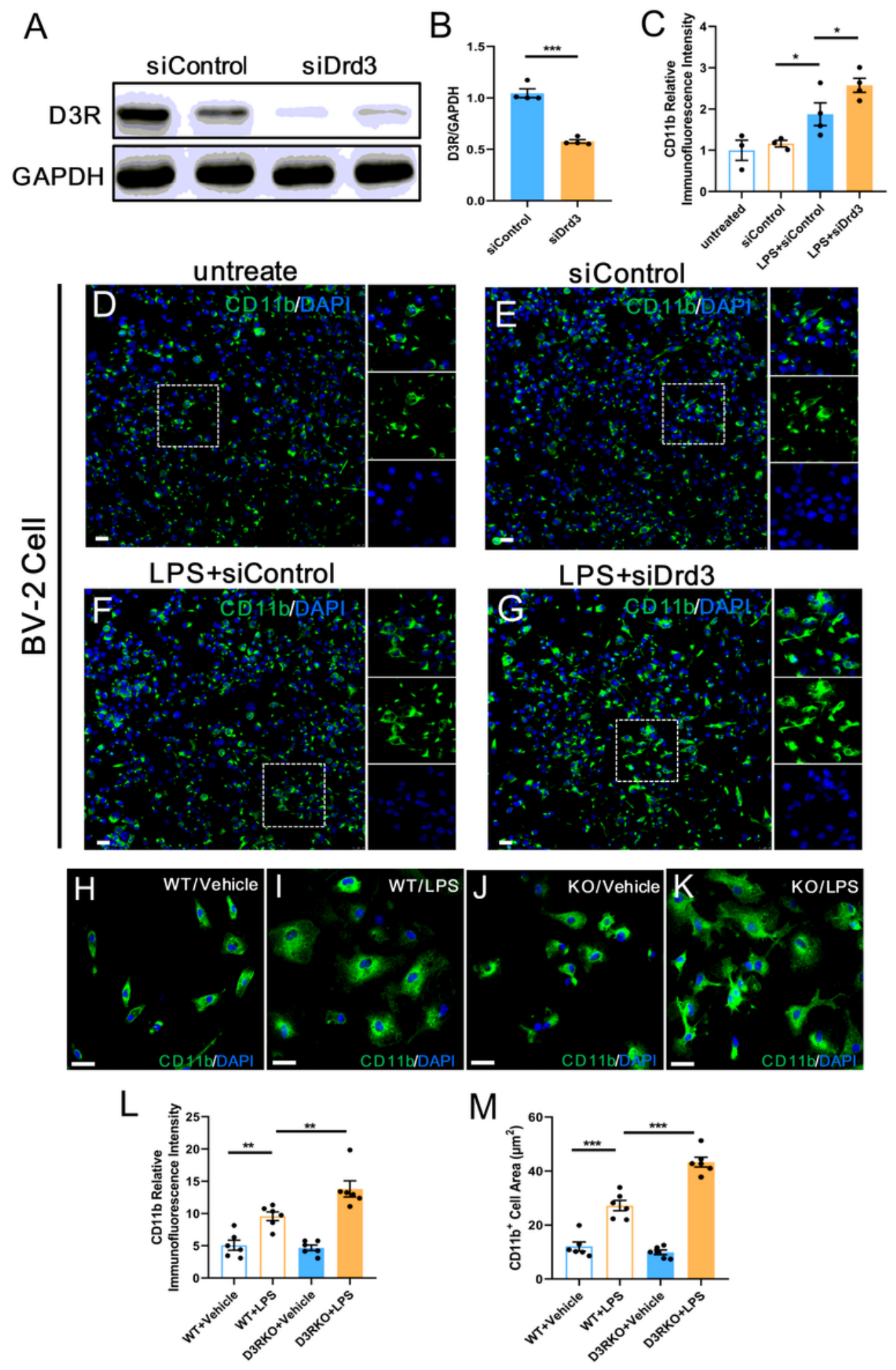

\section{Figure 3}

Microglia activation after down-regulated D3R expression in vitro study. (a-b) In cultured BV-2 cell, western blot analysis showed that D3R expression decreased after using D3R siRNA when compared to control group. (c) Quantitative analysis of CD11b-labelled microglia fluorescence intensity in the cultured BV-2 cells at different groups ( $n=3-4 /$ group). ( $d-g)$ CD 11b-labelled microglia from control BV-2 cells displayed small soma and weak fluorescence intensity without LPS stimulation, but CD11b-labelled 
microglial cell body and immunoreactivity were enhanced after LPS stimulation. Of note, compared to BV-2 cells stimulated with LPS alone, D3R knockdown used by siRNA led to an evident higher intensity of CD11b staining and larger CD11b-labelled microglial cell body in BV-2 cells stimulated with LPS. (scale bar $100 \mu \mathrm{m}$ ). (h-k) In primary cultured microglia, immunostaining results revealed that CD11b-labelled microglia obtained from D3RKO mice had a larger cell body and more ramified process than the corresponding of WT primary microglia cells when subjected to LPS, but there were no obvious alterations in CD11b-marked microglia between WT and D3RKO mice under unstimulated condition (scale bar $50 \mu \mathrm{m})$. (l, $\mathrm{m})$ quantitative analysis of CD11b-labelled microglial fluorescence intensity and cell areas from D3RKO and WT mice with or without LPS stimulation ( $n=6 /$ group). The data are presented as the mean \pm SEM. One-way ANOVA followed by LSD's multiple comparisons post hoc test were used to assess significant differences. ${ }^{\star} \mathrm{p} \rrbracket 0.05,{ }^{*} \mathrm{p} \otimes 0.01,{ }^{* * *} \mathrm{p} \rrbracket 0.001$. 
A

\begin{tabular}{|l|l|l|l|l|l|}
\hline CMV- $\beta$-globin & eGFP \\
\hline
\end{tabular}

B

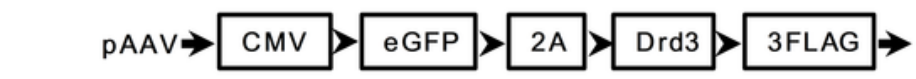

C

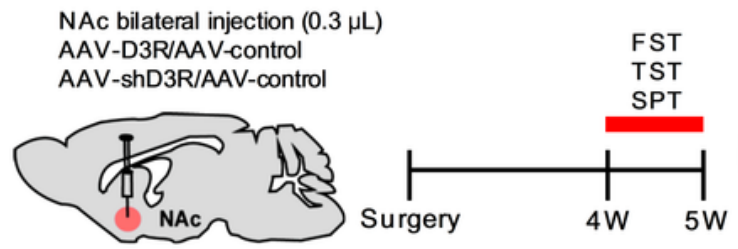

F

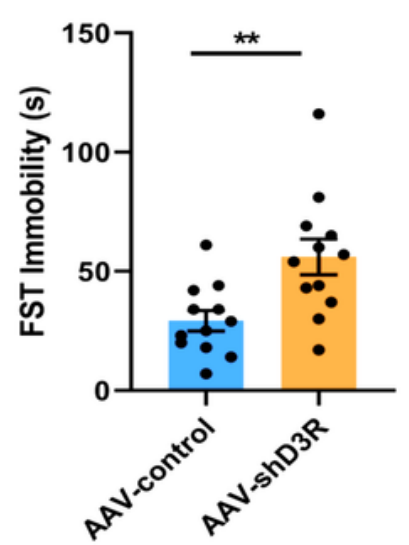

G

J

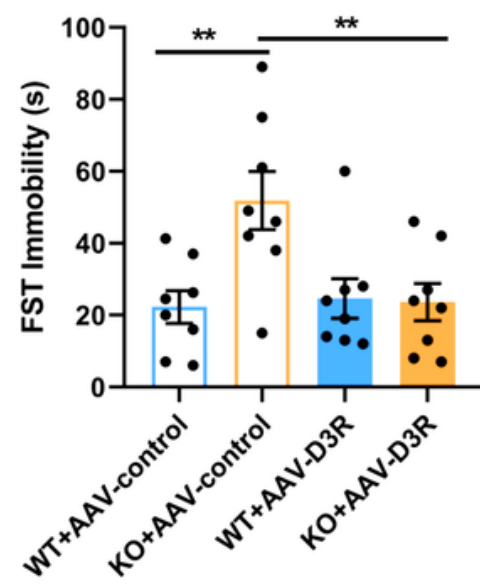

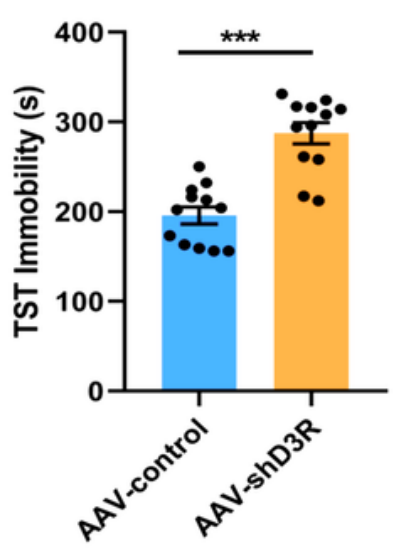

D

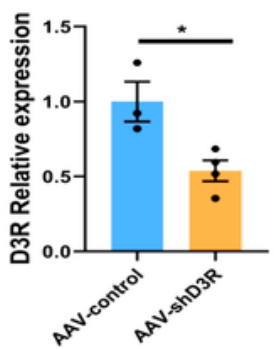

E
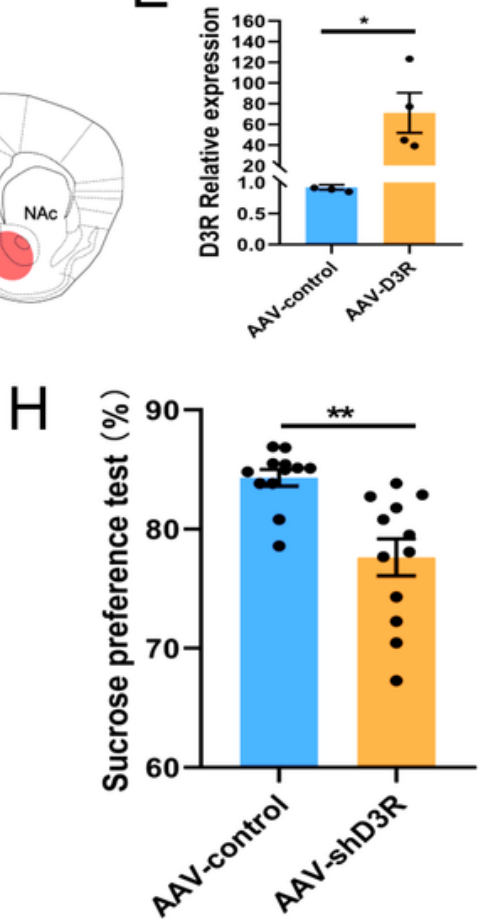

K
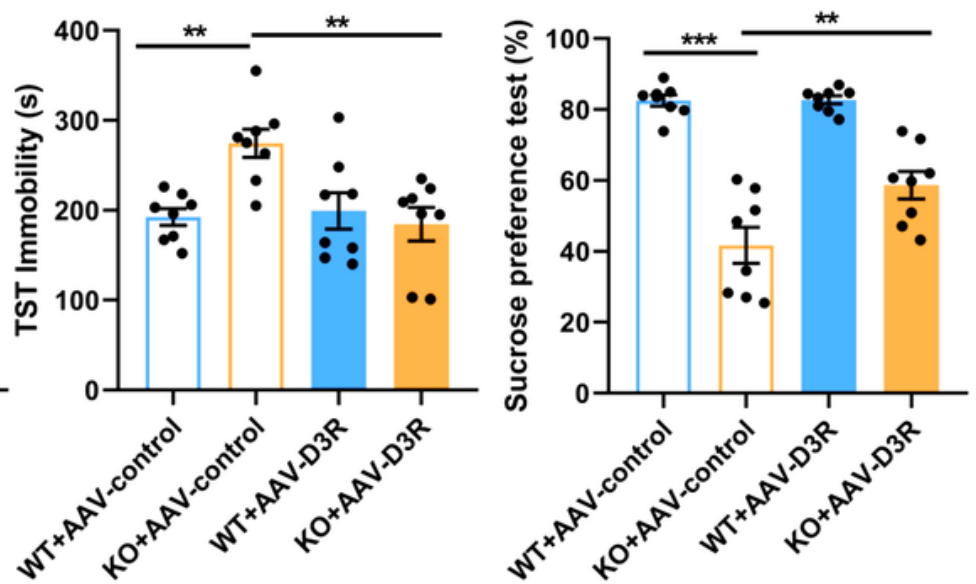

Figure 4

The effects of D3R in the NAc on depressive-like behaviors. (a) Schematic representations of AAV2/8 vector expressing a Drd3-targeting shRNA transcript to adult male mice to make a conditional knockout (CKO) of D3R in the NAc. (b) Schematic representation of AAV2/8-Drd3 vector to adult D3RKO mice to over-express D3R in the NAc. (c) Summary experimental procedure showing the behavioral tests following AAV-shD3R and AAV-D3R injection. (d-e) qRT-PCR analysis confirmed that the expression of 
D3R in the NAc was decreased after using AAV-shD3R and was obviously increased after using AAV-D3R ( $n=3-4 /$ group). ( $f-h)$ Adult mice injected with AAV-shD3R spent more time immobile in the FST and TST, as well as displayed anhedonia as assessed with the sucrose preference test when were comparable to AAV-control mice ( $n=12 /$ group). (i-k) Behavioral tests demonstrated that over-expression of D3R in the NAc were sufficient to rescue the observed depressive-like abnormalities from D3RKO control mice, including the FST, TST and sucrose preference test ( $n=8 /$ group). The data are presented as the mean $\pm S E M$. One-way ANOVA followed by LSD's multiple comparisons post hoc test were used to assess significant differences. ${ }^{*} \mathrm{p} \otimes 0.05,{ }^{*} \mathrm{p} \otimes 0.01,{ }^{* \star *} \mathrm{p} \otimes 0.001, \mathrm{~ns}$ : no significance. 

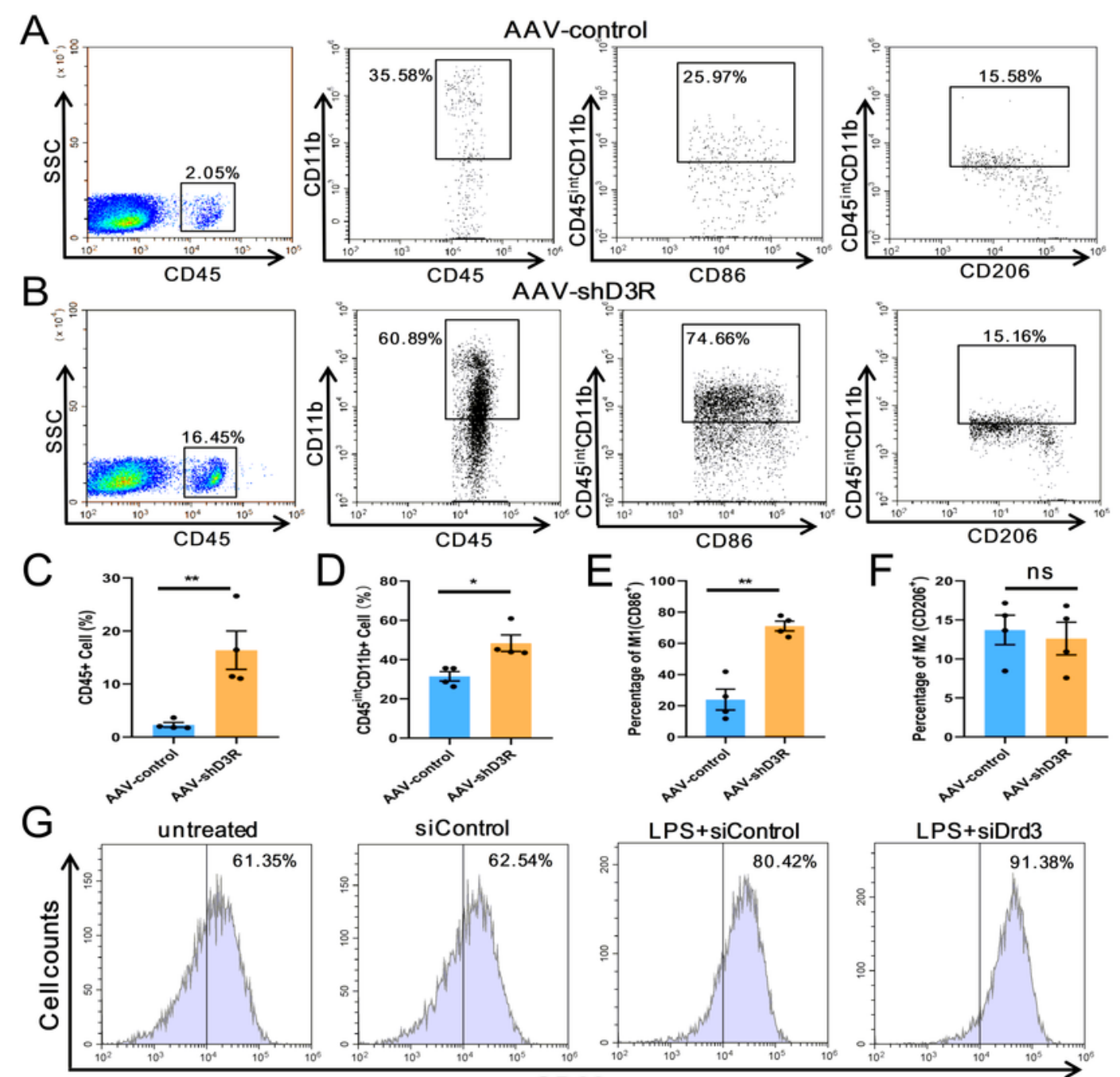

siControl

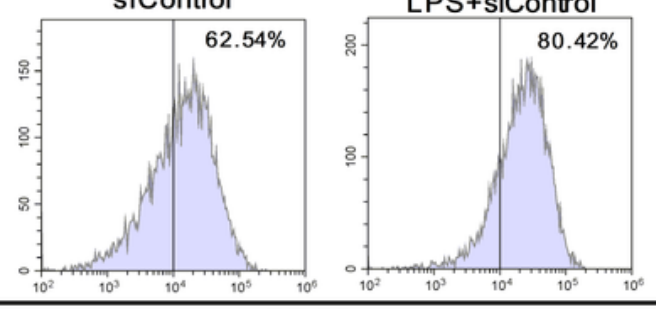

LPS+siDrd3
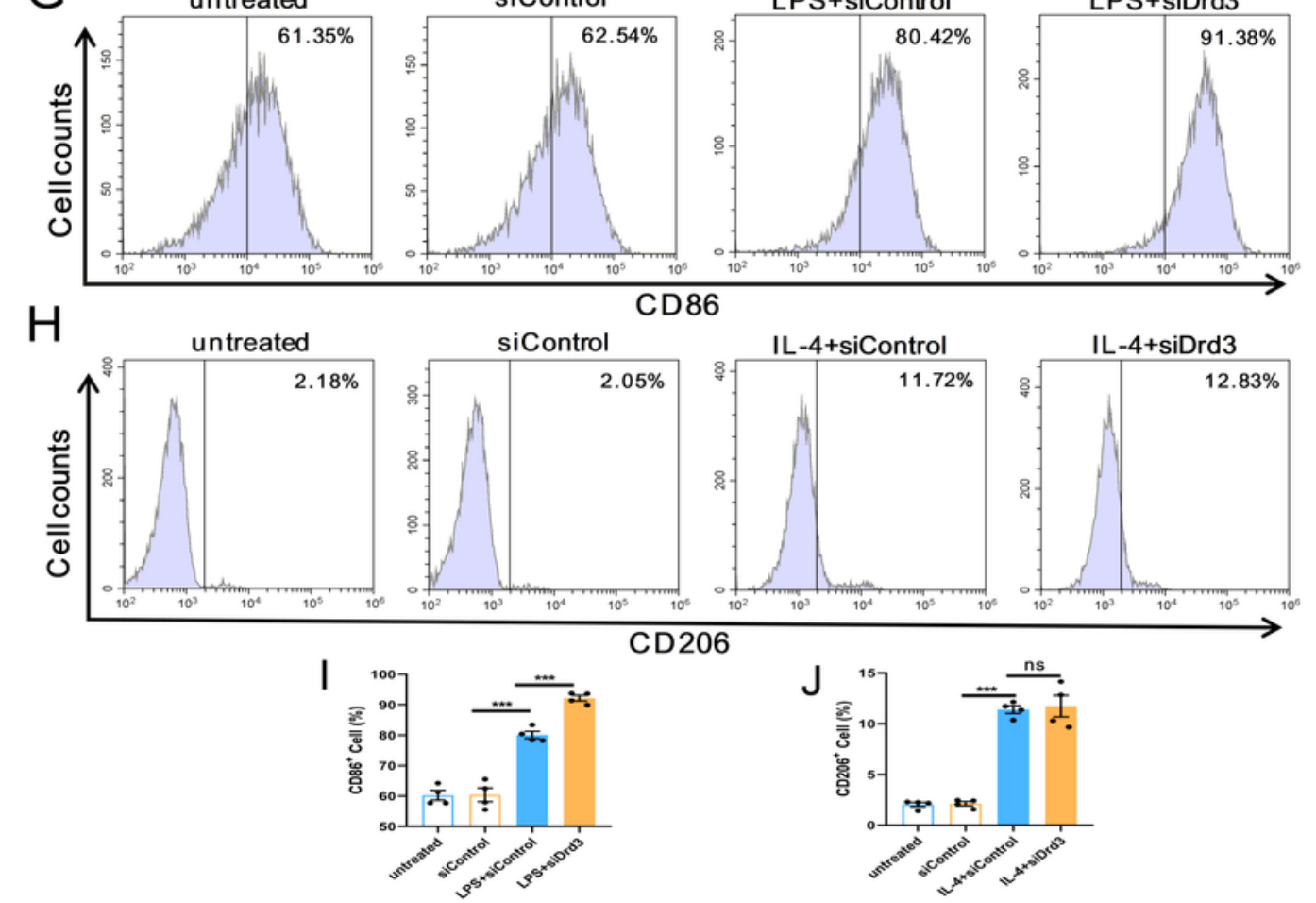

\section{Figure 5}

D3R inhibition in the NAc promotes microglial polarization to M1 phenotype. (a-b) Microglia expression (CD45intCD11b) and microglial M1 phenotype (CD86) in the NAc were markedly in the D3R CKO mice when compared to control group, while microglial M2 phenotype (CD206) in the NAc did not found any difference between between CKO and control mice. (c-f) Summary data for expression of microglia (CD45intCD11b) and microglial M1 (CD86) and M2 (CD206) phenotype in the NAc obtained from D3R 
CKO and control mice ( $\mathrm{n}=4 /$ group). (g) After induction of BV-2 to microglial M1 phenotype by using LPS, suppression of D3R significantly exacerbated CD86 expression, compared to LPS treatment alone. (h) In results of IL-4 treatment-induced microglial M2 phenotype, inhibition of D3R expression did not change IL-4 stimulation-induced CD206 expression in BV-2 microglial cells. (i-j) Summary data for CD86 expression after LPS-induced microglial M1 phenotype and CD206 expression after IL-4 treatmentinduced microglial M2 phenotype ( $n=4 /$ group). The data are presented as the mean $\pm S E M$. One-way ANOVA followed by LSD's multiple comparisons post hoc test were used to assess significant differences. ${ }^{*} \mathrm{p} \llbracket 0.05,{ }^{*} \mathrm{p} \otimes 0.01,{ }^{* \star *} \mathrm{p} \otimes 0.001$, ns: no significance. 

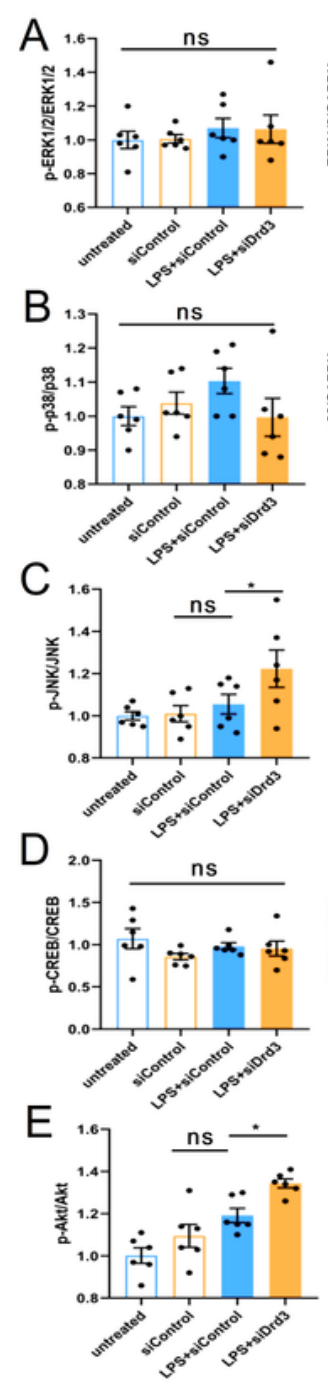

$\mathrm{G}$
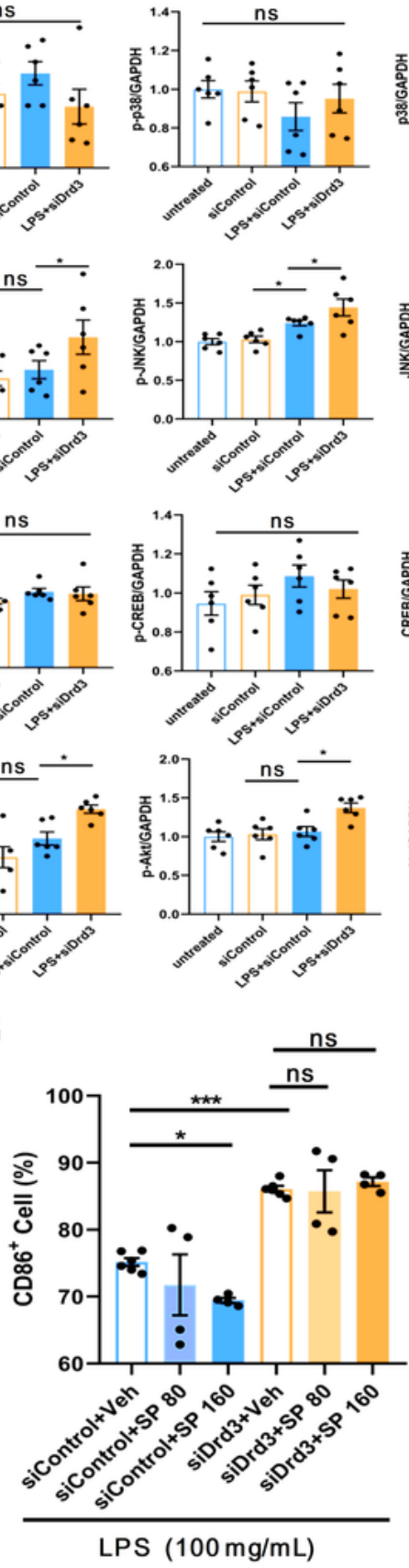

F
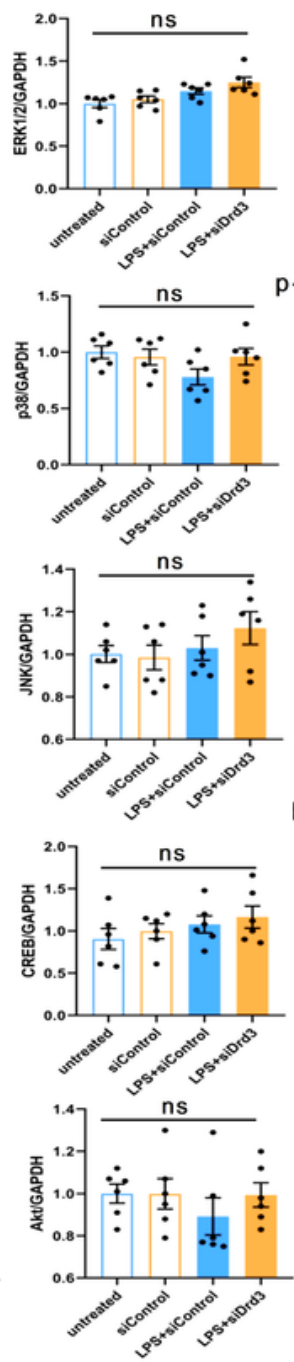

: GAPDH
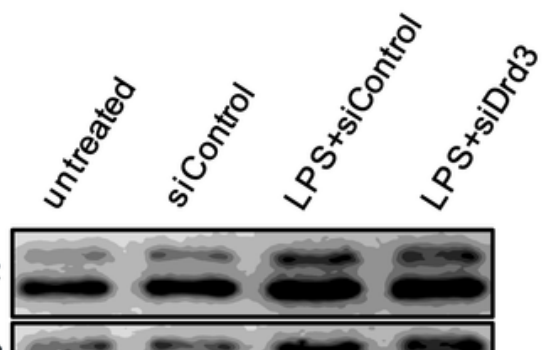

ERK1/2

$p-p 38$
p38

p-JNK

JNK

p-CREB

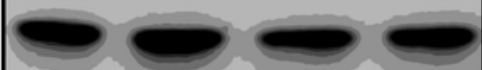

CREB

p-Akt

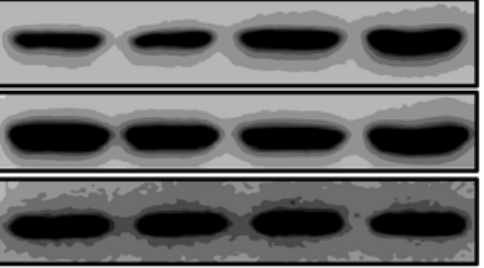

$\mathrm{H}$

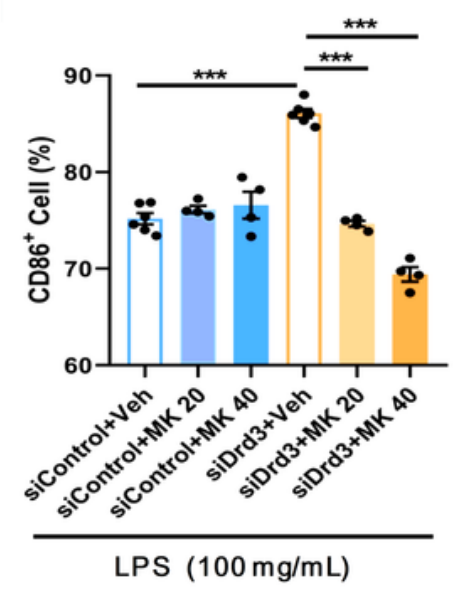

\section{Figure 6}

Effects of Akt signaling pathway on microglia activation induced by D3R inhibition. (a-e) Respective quantitative analysis showing the effects of D3R inhibition on the expression and activation of ERK1/2, p-38, JNK and CREB and Akt signaling pathways after LPS stimulation in the cultured BV-2 cells $(n=6 /$ group). (f) Western blot analysis confirmed the expression alterations in ERK1/2, p-38, JNK and CREB and Akt signaling pathways in BV-2 cells after co-treatment of LPS and D3R inhibition. (g) 
SP600125 pre-treatment (JNK signaling pathway inhibitor) at $80 \mathrm{uM}$ and $160 \mathrm{uM}$ did not show any statistical difference in CD86 expression after stimulated with siDrd3 in BV-2 cells, although $160 \mathrm{uM}$ SP600125 could alleviate CD86 expression increase in BV-2 cells after LPS and siControl treatment ( $n=6 /$ group). (h) In the condition of LPS stimulation, 20 uM and 40 uM MK2206 treatment (Akt signaling pathway inhibitor) dose-dependently attenuated CD86 expression stimulated with siDrd3 in BV-2 cells when compared those to corresponding control group ( $n=6 /$ group). The data are presented as the mean $\pm S E M$. One-way ANOVA followed by LSD's multiple comparisons post hoc test were used to assess significant differences. ${ }^{\star} \mathrm{p} \otimes 0.05,{ }^{*} \mathrm{p} \otimes 0.01,{ }^{\star * \star} \mathrm{p} \otimes 0.001, \mathrm{~ns}$ : no significance.

\section{Supplementary Files}

This is a list of supplementary files associated with this preprint. Click to download.

- SFig.1.tif

- SFig.2.tif

- SFig.3.tif 